\title{
Bifurcation Analysis of a DC-DC Bidirectional Power Converter Operating with Constant Power Loads
}

\author{
Rony Cristiano and Daniel J. Pagano* \\ Department of Automation and Systems, \\ Federal University of Santa Catarina, Florianópolis, Brazil \\ rony.cristiano@ufsc.br,daniel.pagano@ufsc.br \\ Luis Benadero \\ Department of Applied Physics, \\ Technical University of Catalonia, Barcelona, Spain \\ luis@fa.upc.edu \\ Enrique Ponce \\ Department of Applied Mathematics II, \\ University of Seville, Seville, Spain \\ eponcem@us.es
}

\begin{abstract}
Direct current microgrids (MGs) are an emergent option to satisfy new demands for power quality and integration of renewable resources in electrical distribution systems. This work addresses the large-signal stability analysis of a $D C$ - $D C$ bidirectional converter (DBC) connected to a storage device in an islanding direct current (DC) MG. This converter is responsible for controlling the balance of power (load demand and generation) under constant power loads (CPLs). In order to control the $\mathrm{DC}$ bus voltage through a $\mathrm{DBC}$, it is proposed a robust sliding mode control (SMC) based on a washout filter. Dynamical systems techniques are exploited to assess the quality of this switching control strategy. In this sense, a bifurcation analysis is performed to study the nonlinear stability of a reduced model of this system. The appearance of different bifurcations when load parameters and control gains are changed is studied in detail. Both a deeper insight in the dynamic behavior of the controlled system and valuable design criteria are obtained.
\end{abstract}

Keywords: Bifurcation analysis; DPWS systems; bidirectional power converter; sliding mode control; constant power load.

Received (to be inserted by publisher)

\section{Introduction}

Nowadays, DC MGs are an alternative to satisfy new demands of quality energy and integration of renewable resources in electrical distribution systems [Boroyevich et al., 2010; Tahim et al., 2012]. In fact, they

*Author for correspondence 
have been gaining ground on energy distribution in the form of small DC MGs, appearing embedded in several autonomous systems from ships to notebooks. This resumption of DC power distribution is due to the ease of integration of renewable energy resources and the growing share of electronic loads in the system, which makes DC distribution a worthy option to build more efficient systems. Microgrids are local power networks capable of manage energy supply and demand using distributed energy resources (DERs). Most of the time, MGs are connected to the main grid, however they have the ability to operate autonomously in island mode when necessary. These features make the MGs a good solution for the expansion and reliability of the network, paying attention to environmental concerns, since many of DERs are typically renewable sources. Besides, the MG system architecture associated with the new generation of power converters makes the DC distribution more efficient than alternating current (AC) in some contexts, giving rise to DC MGs.

Direct current MGs are composed basically by four elements: (i) a three-phase AC-DC bidirectional power converter to interconnect the microgrid to the main grid by means of an islanding switch; (ii) storage energy devices (batteries, fly-wheels, etc.); (iii) renewable energy resources (wind turbine generators (WTGs), photovoltaic panels (PVs), hydrogen fuel-cell, etc.), and (iv) resistive, constant current and CPLs. A typical DC microgrid is depicted in Fig. 1.

When the microgrid is in island mode operation, the load power demand must be provided only by the DERs and storage devices connected to the DC bus. In this context, the DC bus voltage must be stabilized also assuring a correct balance between the power generated by the DERs and the load power demand. This balancing goal generally is assigned to storage devices as a battery connected to the DC bus through a bidirectional converter, as depicted in Fig. 1. When the DERs generate more power than the load needs, the $D C-D C$ bidirectional converter (DBC) connected to the battery must transfer the excess of available power to the battery, storing it. When loads need more power than available from the sources, the $\mathrm{DBC}$ is responsible to return the stored energy in the battery to achieve the power balance. Thus, the DBC connected to the battery has two main goals when the microgrid is isolated from the main grid (i) to control the power flow of batteries and (ii) to keep the DC bus voltage constant, even driving nonlinear loads as is the case of CPLs.

This work addresses the nonlinear stability analysis of the DC link bus voltage taking into account that the equivalent load as seen from the DBC is nonlinear and assuming a CPL behavior. This analysis gives the background to understand the proposed SMC based on a washout filter, which is designed to regulate the DC bus voltage under nonlinear load variations. The SMC is responsible for controlling the balance of power (load power demand and power generation) through a DBC. This converter is connected to a storage device that will allow to maintain, under an adequate control strategy, a constant DC bus voltage of the DC microgrid operating in islanding mode.

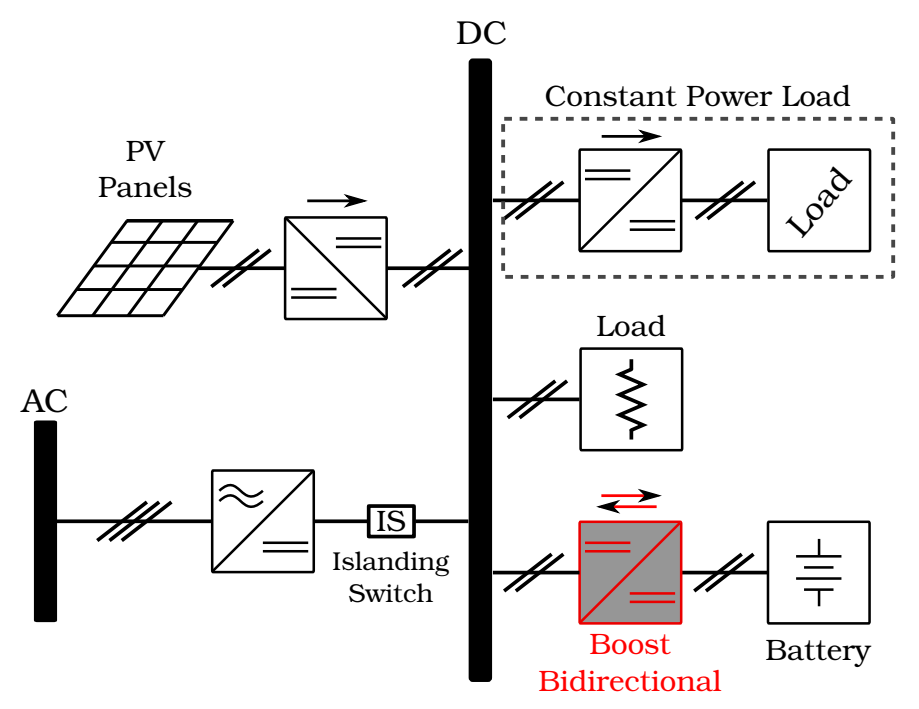

Figure 1. Typical DC microgrid with a DBC (boost bidirectional) connected to a battery. 
Power converters interconnection topologies based on a cascade structure are studied in [Haroun, 2014, 2015]. A systematic procedure to synthesize cascade connection of DC-DC boost converters operating with sliding mode control is presented in [Haroun, 2014]. Moreover, this method is applied to a system connected to a photovoltaic source consisting of two cascaded DC-DC boost converters under sliding mode control in [Haroun, 2015].

Other some related works on this subject have been reported in the literature. For instance, a detailed Bifurcation analysis of a single boost controlled by a SMC can be found in [Ponce \& Pagano, 2011]. Smooth and non-smooth bifurcations in multi-structure multi-operating-mode hybrid power systems are studied in [Xiong et al., 2013b] where a hybrid power system consisting of dual-input buck converters is analysed. Also in [Xiong et al., 2013a], the same authors showed a bifurcation analysis based on averaged models for a stand-alone photovoltaic-battery hybrid power system. Modeling DC microgrids in stand-alone operation can be found in [Tahim et al., 2012].

The main contribution of this paper is the proposal of a rigorous methodology for the obtention of a complete bifurcation analysis regarding a bidirectional power converter operating in a stand alone DC microgrid. We show the existence of new bifurcations (boundary equilibrium bifurcations and the T-singularity bifurcation) induced by the discontinuity in this class of systems controlled by SMC. Once completed the bifurcation set, the parameter regions with different possible behaviors are identified, getting so a valuable tool for a deeper and sound design and control. In particular, we show the "safe" regions for choosing the adequate values of parameters to be located sufficiently far from destabilizing bifurcations.

The paper is organized as follows. Section 2 describes the model of the bidirectional converter and the proposed SMC. The theory of discontinuous piecewise smooth (DPWS) systems is reviewed in Section 3. A detailed analysis of bifurcations taking into account the load parameters and the controller gain is presented in Sections 4 and 5.

\section{DC microgrid modeling and control}

The DC microgrid studied in this work is depicted in Fig. 2. This DC MG operates in the islanding mode (stand-alone operation), i.e. without connection to a main feed grid. In this case study, there are two main energy sources (i) a PV source connected to the DC by means of a DC-DC power converter operating in maximum power point tracking (MPPT) mode; and (ii) a battery pack. The PV source behaves like a power source, injecting constant power to the microgrid, and thus it can be considered as a constant power sources (CPSs) denoted by $P_{S}$. This source is dependent on the weather conditions, but for steady climate conditions this modeling approach is accurate. The battery can be also regarded as an ideal DC voltage source $\left(V_{i n}\right)$ since its dynamic is very slow. In this way, assuming the above hypothesis, the dynamic behaviors of these two sources are not taken into account in this work. Furthermore, a DC architecture is based on multistage power converters where point-of-load (POL) converters can be seen by the feeder converter as CPLs, namely $P_{L}$ [Emadi et al., 2006; Liutanakul et al., 2010; Kwasinski et al., 2011]. Fig. 2 shows a block diagram of this reduced equivalent model.

The generated and consumed power can be modeled as $P=P_{S}+P_{L}$, where it is assumed $P_{S}<0$ and $P_{L}>0$ leading to a simplified circuit showed in Fig. 3. There the balance of power between generation and load demanding is controlled by a bidirectional converter using a battery to drain or inject energy into the MG.

From the simplified model showed in Fig. 3, the DC bus current and the equivalent load $\left(R_{e q}\right)$ are given by

$$
i_{\text {bus }}=\frac{v_{c}}{R}+\underbrace{\frac{P}{v_{c}}}_{\mathrm{CPL}} \text { and } R_{e q}=\frac{v_{c}}{i_{\text {bus }}},
$$

where $v_{c}$ is the capacitor voltage. From (1), a $v_{c}-i_{\text {bus }}$ characteristics of each type of load can be obtained as depicted in Fig. 4, where the arrows indicate the direction of resistive load reduction. The incremental impedance $\left(d v_{c} / d i_{b u s}\right)$ is always positive when $P \leq 0$, that is, a decrease (increase) in the voltage results in a decrease (increase) in the current. This situation occurs if the power generated is equal or greater than 


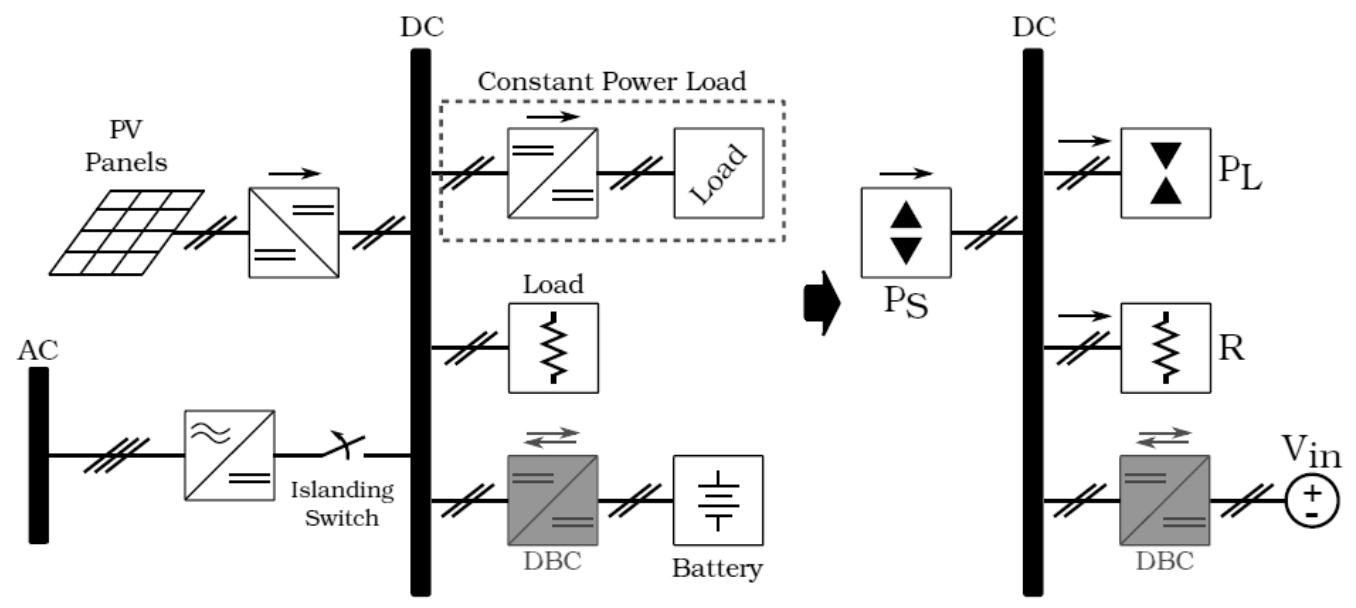

Figure 2. DC MG and its equivalent model in stand-alone operation.

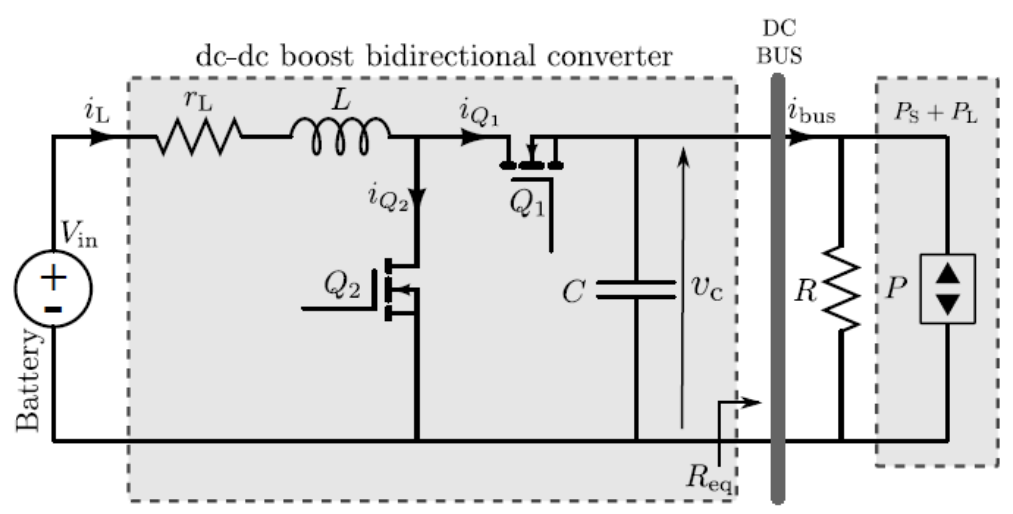

Figure 3. DC MG simplified model in stand-alone operation.

the CPLs power demand. When $\left|P_{S}\right|<P_{L}$, that is $P>0$, although the instantaneous impedance is always positive $\left(R_{e q}>0\right)$, the incremental impedance may be negative $\left(d v_{c} / d i_{\text {bus }}<0\right)$ leading to an unstable behavior under small disturbances in current or voltage [Emadi et al., 2006]. Thus, a critical situation arises when the available power from the sources is not enough to supply the CPL demand.

The circuit showed in Fig. 3 is modeled assuming that the two switches $Q_{1}$ and $Q_{2}$ are complementary, that is when $Q_{1}$ is driven, $Q_{2}$ is blocked and viceversa. In this way, defining $u \in\{0,1\}$ as a two-state control variable, a simplified model of the DC microgrid can be represented as

$$
\begin{aligned}
L \frac{d i_{L}}{d t} & =V_{i n}-r_{L} i_{L}-u v_{c} \\
C \frac{d v_{c}}{d t} & =u i_{L}-\frac{v_{c}}{R}-\frac{P}{v_{c}}
\end{aligned}
$$

where $v_{c}>0$ and $i_{L}$ stand for the dc voltage and the inductor current, respectively, and will be assumed as the state variables; $V_{i n}$ is the ideal voltage source from battery, $R$ is the total load resistance on the bus, $r_{L}$ is the inductor resistance (we assume a non ideal inductance). For simplicity, the MG transmission line impedance is not considered in this work assuming that the distances between the MG devices are very short.

As in [Tahim et al., 2012], equations (2)-(3) can be normalized applying the change of variables and 


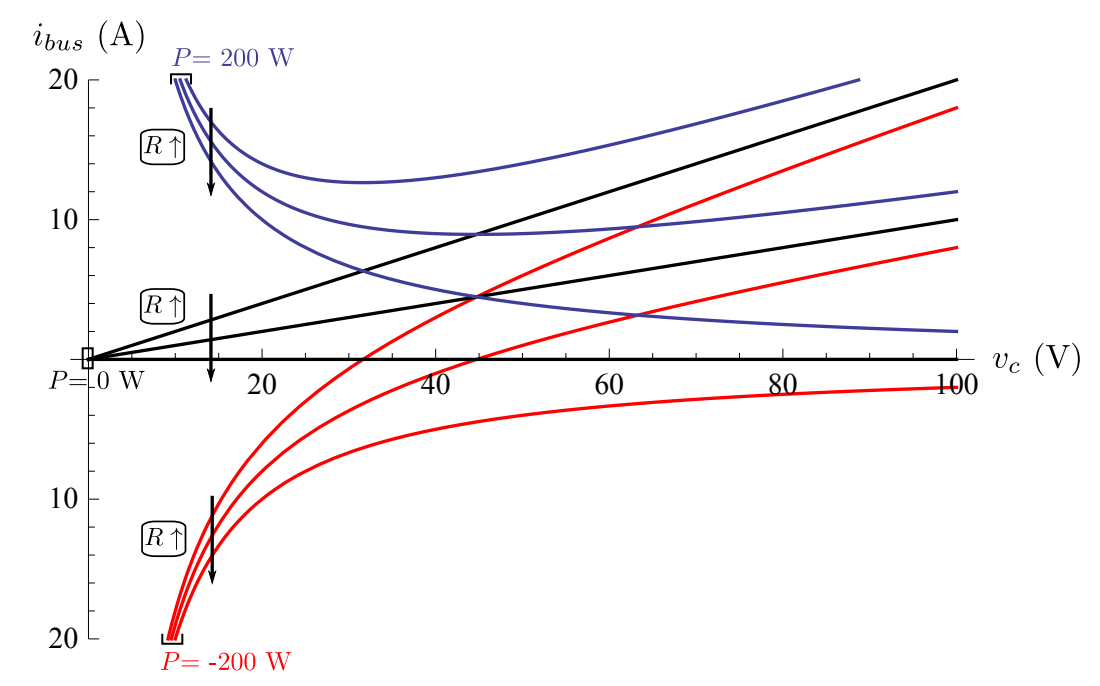

Figure 4. $\left(v_{c}, i_{\text {bus }}\right)$ characteristics for several values of the parameters $P$ and $R$.

time

$$
i_{L}=\sqrt{\frac{C}{L}} V_{i n} x, \quad v_{c}=V_{i n} y, \quad t=\tau \sqrt{L C} .
$$

We define the new parameters

$$
a=\frac{1}{R} \sqrt{\frac{L}{C}}, \quad b=r_{L} \sqrt{\frac{C}{L}}, \quad d=\frac{P}{V_{i n}^{2}} \sqrt{\frac{L}{C}},
$$

in such a way that the dimensionless model can be written as

$$
\begin{aligned}
& \frac{d x}{d \tau}=1-b x-u y \\
& \frac{d y}{d \tau}=u x-a y-\frac{d}{y},
\end{aligned}
$$

where $a>0, b>0$ and $d \in \mathbb{R}$. The new state variables are now $x \in \mathbb{R}$ and $y>0$. Parameters $a$ and $d$ stand for the normalized resistive and power loads of the circuit system, respectively and will be considered as the main bifurcation parameters. Clearly, these parameters vary with the power demand and the availability of sources on the MG, thus causing changes in the system dynamics and uncertainty on the location of the desired operating point in the state space.

The control objectives are basically (i) to regulate the desired value of the output voltage $y=y_{r}$, where $y_{r}>1$, in steady state; (ii) to ensure robustness of the system to parameter $a$ and $d$ variations, produced mainly by load and power demand changes; (iii) to minimize the transient response when $a$ and $d$ varies.

A SMC strategy based on a washout filter (high-pass filter) is adopted to control this system [Tahim et al., 2012], [Pagano et al., 2013]. A washout filter is a high-pass linear filter that washes out steady-state inputs while passing transient inputs ([Lee \& Abed, 1991], [Wang \& Abed, 1995]). We assume that the inductor current $x$ can be filtered to get a new signal $x_{F}$, and that the transfer function of the washout filter is

$$
G_{F}(s)=\frac{X_{F}(s)}{X(s)}=\frac{s}{s+w}=1-\frac{w}{s+w},
$$

where $w$ denotes the reciprocal of the filter constant and $x_{F}$ is the filter output.

The washout filter together with an appropriate choice of the switching surface eliminates the dependence of the voltage of pseudo-equilibrium point (operating point for the converter) with respect to the parameters $a$ and $d$ [Ponce \& Pagano, 2011]. The effect of the washout filter can be represented by means of an additional differential equation, namely $\dot{z}=w(x-z)$, being $w>0$ a new parameter to be tuned and $z$ a new state variable that satisfies the output equation $x_{F}=x-z$. 


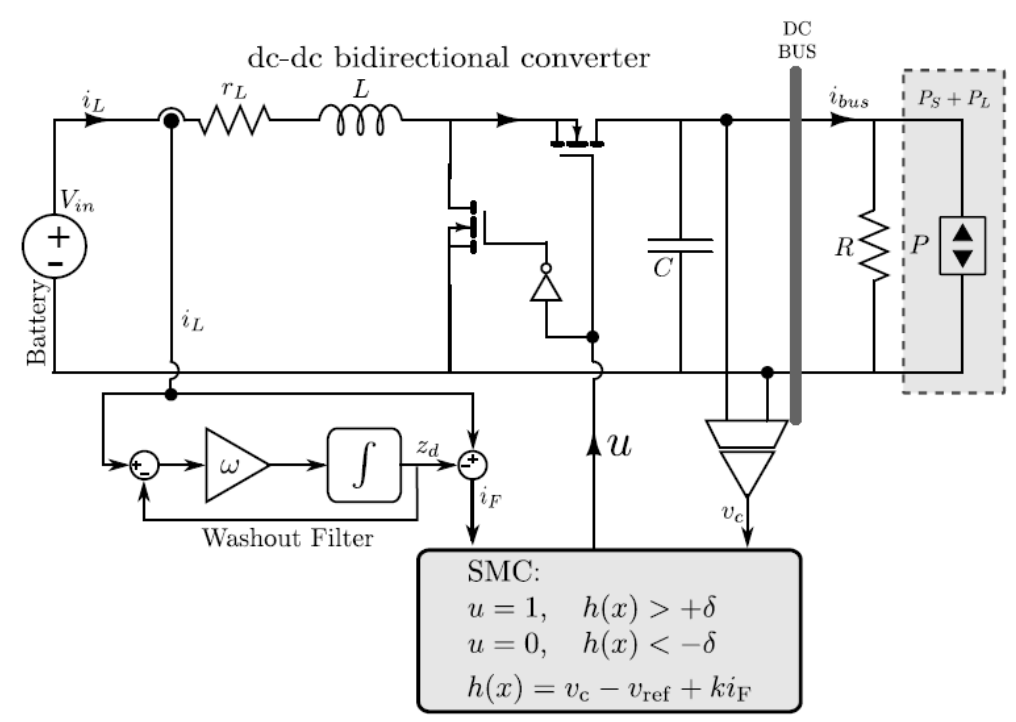

Figure 5. Schematic diagram of the DBC circuit with SMC.

We define the manifold $\Sigma=\left\{\mathbf{x} \in \mathbb{R}^{3}: h(\mathbf{x})=0\right\}$ through the scalar function $h: \mathbb{R}^{3} \rightarrow \mathbb{R}$ with

$$
h(\mathbf{x})=y-y_{r}+k(x-z),
$$

where $y_{r}$ is the desired normalized voltage at the operating point and $k>0$ is the control parameter that must be adjusted to guarantee system stability and transients specifications. The SMC law is then defined as

$$
u=\frac{1}{2}(1+\operatorname{sign} h(\mathbf{x}))
$$

with $\mathbf{x}=(x, y, z) \in \mathbb{R}^{3}$.

Note that the output $x_{F}=x-z$ of the washout filter is used in equation of $h(\mathbf{x})$ and that it vanishes in steady state. Therefore from (6), the voltage of the pseudo-equilibria are not affected by load changes. A schematic diagram of the DBC circuit with the proposed SMC is shown in Fig. 5.

The dynamics of the system, using the proposed SMC control with washout filter can be represented as a dynamical DPWS system

$$
\dot{\mathbf{x}}= \begin{cases}\mathbf{F}^{-}(\mathbf{x}), & \text { if } h(\mathbf{x})<0 \\ \mathbf{F}^{+}(\mathbf{x}), & \text { if } h(\mathbf{x})>0\end{cases}
$$

composed by the vector fields

$$
\mathbf{F}^{-}(\mathbf{x})=\left[\begin{array}{c}
1-b x \\
-a y-\frac{d}{y} \\
w(x-z)
\end{array}\right] \text { and } \mathbf{F}^{+}(\mathbf{x})=\left[\begin{array}{c}
1-b x-y \\
x-a y-\frac{d}{y} \\
w(x-z)
\end{array}\right] .
$$

A review of the basic theory on DPWS systems will be addressed in the next Section in order to facilitate the subsequent dynamical analysis of system (8).

\section{DPWS Systems}

Switching electronic power converters can be considered as DPWS systems, also named in the literature as Filippov systems [Filippov, 1988; Kuznetsov et al., 2003]. In what follows we review succinctly the theory of DPWS systems. 
Let us assume two vector fields $\mathbf{F}^{ \pm}: \mathbb{R}^{n} \rightarrow \mathbb{R}^{n}$ and a scalar function $h: \mathbb{R}^{n} \rightarrow \mathbb{R}$, such that 0 is one of its regular values and so the set $\Sigma=\left\{\mathbf{x} \in \mathbb{R}^{n}, h(\mathbf{x})=0\right\}$ is a smooth manifold with non-vanishing gradient $h_{\mathrm{x}}$ on it. This manifold is chosen to be a switching boundary, splitting the phase space into two open regions $R^{-}=\left\{\mathbf{x} \in \mathbb{R}^{n}: h(\mathbf{x})<0\right\}$ and $R^{+}=\left\{\mathbf{x} \in \mathbb{R}^{n}: h(\mathbf{x})>0\right\}$. A DPWS dynamical system is defined as

$$
\dot{\mathbf{x}}= \begin{cases}\mathbf{F}^{-}(\mathbf{x}), & \text { if } \mathbf{x} \in R^{-} \\ \mathbf{F}^{+}(\mathbf{x}), & \text { if } \mathbf{x} \in R^{+},\end{cases}
$$

where the vector field should be appropriately extended to $\Sigma$ following Filippov's convention [Kuznetsov et al., 2003]. On the switching boundary $\Sigma$, three different regions can be defined (see Fig. 6): the crossing (sewing), the attractive sliding and the repulsive sliding (escaping). These regions are determined using the two orbital derivatives of $h$ or Lie derivatives $L_{\mathbf{F}^{ \pm}} h=h_{\mathbf{x}} \cdot \mathbf{F}^{ \pm}$, where $h_{\mathbf{x}}$ is the gradient of $h$.

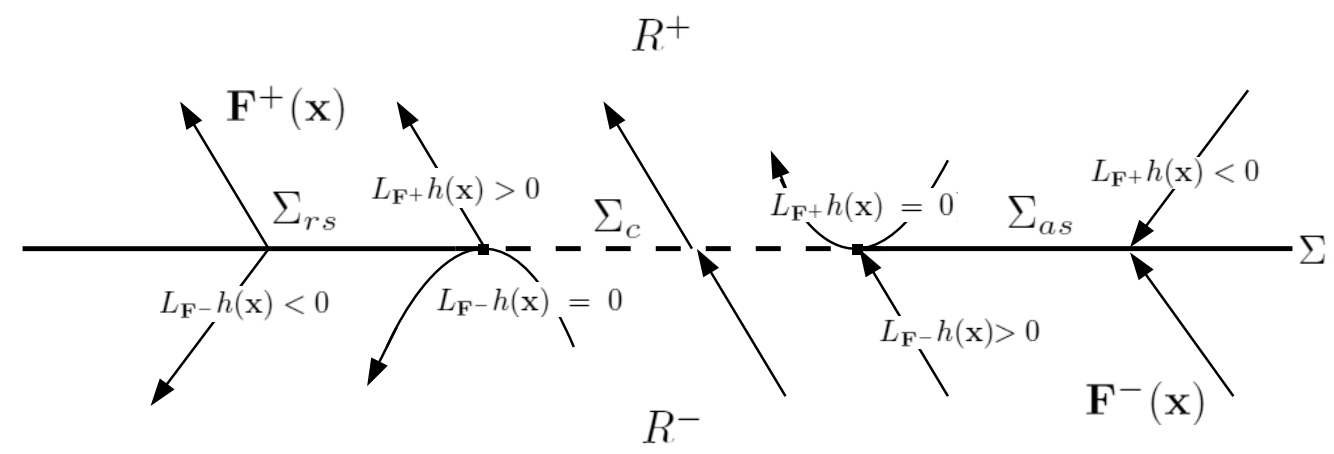

Figure 6. Typical regions on $\Sigma$ in $\mathbb{R}^{2}$ showing the escaping, crossing and attractive sliding parts.

The attractive sliding region is defined as

$$
\Sigma_{a s}=\left\{\mathbf{x} \in \Sigma: L_{\mathbf{F}^{+}} h(\mathbf{x})<0<L_{\mathbf{F}^{-}} h(\mathbf{x})\right\},
$$

where the vector fields $\mathbf{F}^{ \pm}$point to the surface on both sides. The repulsive sliding region is defined as

$$
\Sigma_{r s}=\left\{\mathbf{x} \in \Sigma: L_{\mathbf{F}^{-}} h(\mathbf{x})<0<L_{\mathbf{F}^{+}} h(\mathbf{x})\right\},
$$

where $\mathbf{F}^{ \pm}$point away from the surface on both sides. The crossing region is given by

$$
\Sigma_{c}=\left\{\mathbf{x} \in \Sigma: L_{\mathbf{F}^{-}} h(\mathbf{x}) \cdot L_{\mathbf{F}^{+}} h(\mathbf{x})>0\right\},
$$

where one of the vector fields points to the surface and the other pointing outwards from the surface.

In the sliding set $\Sigma_{a s} \cup \Sigma_{r s}$, the dynamics is described by the sliding vector field $\mathbf{F}_{s}$ [Kuznetsov et al., 2003], obtained from a convex linear combination of the two vector fields $\mathbf{F}^{ \pm}(\mathbf{x})$ as

$$
\mathbf{F}_{s}(\mathbf{x})=(1-\lambda) \mathbf{F}^{-}(\mathbf{x})+\lambda \mathbf{F}^{+}(\mathbf{x}),
$$

where for each $\mathbf{x} \in \Sigma_{a s} \cup \Sigma_{r s}$, the value of $\lambda$ is chosen such that $L_{\mathbf{F}_{s}} h(\mathbf{x})=0$, i.e.,

$$
\lambda=\lambda(\mathbf{x})=\frac{L_{\mathbf{F}^{-}} h(\mathbf{x})}{L_{\mathbf{F}^{-}} h(\mathbf{x})-L_{\mathbf{F}^{+}} h(\mathbf{x})},
$$

provided that the above denominator does not vanish. Therefore, $\lambda \in(0,1) \forall \mathbf{x} \in \Sigma_{a s} \cup \Sigma_{r s}$, while $\lambda=0$ implies that $L_{\mathbf{F}^{-}} h(\mathbf{x})=0$, i.e., $\mathbf{x}$ is a tangency point of the vector field $\mathbf{F}^{-}$with the boundary $\Sigma$, and $\lambda=1$ implies that $L_{\mathbf{F}^{+}} h(\mathbf{x})=0$, i.e., $\mathbf{x}$ is a tangency point of the vector field $\mathbf{F}^{+}$with the boundary $\Sigma$.

A double tangency is a point where both vector fields $\mathbf{F}^{ \pm}$are tangent to $\Sigma$ [Teixeira, 1990]. The tangency points are the boundaries between the sliding and crossing regions or between crossing regions. In this paper, we focus on the tangency of fold type, for $n \geq 3$, that is a quadratic tangency. Such tangencies can be of two types: visible or invisible. This visibility character is very relevant at these points where the two vector fields involved have a common tangency point. 
Definition 1. A point $\widehat{\mathbf{x}} \in \Sigma$ is called two-fold if $L_{\mathbf{F}^{-}} h(\widehat{\mathbf{x}})=L_{\mathbf{F}^{+}} h(\widehat{\mathbf{x}})=0, L_{\mathbf{F}^{-}}^{2} h(\widehat{\mathbf{x}}) \neq 0$ and $L_{\mathbf{F}^{+}}^{2} h(\widehat{\mathbf{x}}) \neq 0$, involving the simultaneous tangency of $\mathbf{F}^{ \pm}$with $\Sigma$. Furthermore, the sets $S^{-}=\left\{\mathbf{x} \in \Sigma: L_{\mathbf{F}^{-}} h(\mathbf{x})=0\right\}$ and $S^{+}=\left\{\mathbf{x} \in \Sigma: L_{\mathbf{F}^{+}} h(\mathbf{x})=0\right\}$ transversely intersect at $\widehat{\mathbf{x}}$, i.e., the matrix

$$
Q=\left[\begin{array}{c}
\left(L_{\mathbf{F}^{+}} h(\widehat{\mathbf{x}})\right)_{\mathbf{x}} \\
\left(L_{\mathbf{F}^{-}} h(\widehat{\mathbf{x}})\right)_{\mathbf{x}} \\
h_{\mathbf{x}}(\widehat{\mathbf{x}})
\end{array}\right],
$$

has maximal rank. The two-fold can be classified in

- invisible, if $L_{\mathbf{F}^{+}}^{2} h(\widehat{\mathbf{x}})<0<L_{\mathbf{F}^{-}}^{2} h(\widehat{\mathbf{x}})$, i.e. both tangencies are invisible;

- visible, if $L_{\mathbf{F}^{-}}^{2} h(\widehat{\mathbf{x}})<0<L_{\mathbf{F}^{+}}^{2} h(\widehat{\mathbf{x}})$, i.e. both tangencies are visible.;

- visible-invisible, if $L_{\mathbf{F}^{+}}^{2} h(\widehat{\mathbf{x}}) \cdot L_{\mathbf{F}^{-}}^{2} h(\widehat{\mathbf{x}})>0$, i.e. one tangent is visible and the other is invisible.

Remark 1. In Definition 1,

$$
L_{\mathbf{F}^{ \pm}}^{2} h(\widehat{\mathbf{x}})=L_{\mathbf{F}^{ \pm}}\left(L_{\mathbf{F}^{ \pm}} h\right)(\widehat{\mathbf{x}})
$$

and

$$
\left(L_{\mathbf{F}^{ \pm}} h(\widehat{\mathbf{x}})\right)_{\mathbf{x}}=h_{\mathbf{x}}(\widehat{\mathbf{x}}) \cdot \mathbf{F}_{\mathbf{x}}^{ \pm}(\widehat{\mathbf{x}})+\left(\mathbf{F}^{ \pm}(\widehat{\mathbf{x}})\right)^{T} \cdot h_{\mathbf{x x}}(\widehat{\mathbf{x}}),
$$

where $\mathbf{F}_{\mathbf{x}}^{ \pm}$is the jacobian matrix of $\mathbf{F}^{ \pm}$and $h_{\mathbf{x} \mathbf{x}}$ is the Hessian matrix of $h$.

The case of an invisible two-fold in $\mathbb{R}^{3}$, that is the more relevant case, is illustrated in Fig. 7 .

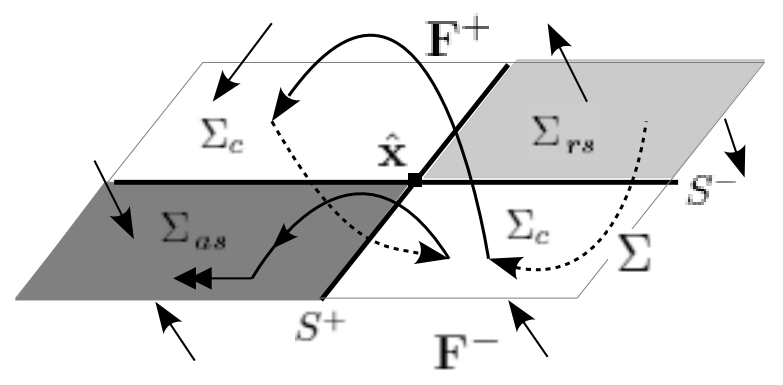

Figure 7. Invisible two-fold in $\mathbb{R}^{3}$.

Remark 2. The invisible two-fold, namely Teixeira singularity [Teixeira, 1990], will be denoted in this work as T-singularity (TS). $\mathbf{F}_{s}$.

System (9) can have equilibria for each vector field $\mathbf{F}^{ \pm}$and also equilibria for the sliding vector field

Definition 2. Let $\overline{\mathbf{x}} \in \mathbb{R}^{n}$ be an equilibrium point of the vector field $\mathbf{F}^{-}\left(\mathbf{F}^{+}\right)$, i.e., $\mathbf{F}^{-}(\overline{\mathbf{x}})=\mathbf{0}\left(\mathbf{F}^{+}(\overline{\mathbf{x}})=\mathbf{0}\right)$. This point is a real equilibrium of $\mathbf{F}^{-}\left(F^{+}\right)$if $h(\overline{\mathbf{x}})<0(h(\overline{\mathbf{x}})>0)$; a virtual equilibrium point if $h(\overline{\mathbf{x}})>0$ $(h(\overline{\mathbf{x}})<0)$; a boundary equilibrium if $h(\overline{\mathbf{x}})=0$.

Boundary equilibrium points represent intermediate situations between real and virtual equilibrium points. Possible topological changes from real to virtual equilibrium points (or viceversa) due to changes in some parameter of the system can lead to boundary equilibrium bifurcations (BEB) [di Bernardo et al., 2008a,b; Pagano et al., 2011].

Definition 3. A point $\widetilde{\mathbf{x}} \in \mathbb{R}^{n}$ is a pseudo-equilibrium of dynamical system (9) if $\widetilde{\mathbf{x}} \in \Sigma$ and is an equilibrium of the sliding vector field $\mathbf{F}_{s}$ given in (13), that is,

$$
\begin{aligned}
(1-\lambda(\widetilde{\mathbf{x}})) \mathbf{F}^{-}(\widetilde{\mathbf{x}})+\lambda(\widetilde{\mathbf{x}}) \mathbf{F}^{+}(\widetilde{\mathbf{x}}) & =\mathbf{0} \\
h(\widetilde{\mathbf{x}}) & =0
\end{aligned}
$$


Moreover, $\widetilde{\mathbf{x}}$ is a real pseudo-equilibrium whenever $\lambda \in(0,1)$, and is a virtual pseudo-equilibrium point provided that $\lambda<0$ or $\lambda>1$.

Remark 3. In the critical cases, given by $\lambda=0$ or $\lambda=1$, we have for sure a collision between a pseudoequilibrium point and a boundary equilibrium point.

In presence of an invisible two-fold point or TS and considering the variation of some parameters of the system, say $\mu \in \mathbb{R}$, there exists also the possibility of a transition of one pseudo-equilibrium point between two regions $\Sigma_{a s}$ and $\Sigma_{r s}$ passing through the TS point. For instance, assume that at the critical value $\mu=\mu_{c}$, a pseudo-equilibrium point collides with the invisible two-fold, so that for $\mu<\mu_{c}$ the pseudoequilibrium point is on $\Sigma_{a s}$ and for $\mu>\mu_{c}$ it is on $\Sigma_{r s}$. In this scenario, it is possible the occurrence of a bifurcation given rise to a closed periodic orbit that crosses the boundary $\Sigma$ using two different portions of $\Sigma_{c}$ (see Fig. 7). Such periodic orbit will be called as crossing limit cycle (CLC). The appearance of this CLC due to the transition of a pseudo-equilibrium point through the TS will be called TS bifurcation [Colombo et al., 2010; Jeffrey \& Colombo, 2009].

\section{Bifurcation analysis}

In this Section, we use the previous results presented in Section 3 to analyse system (8). We begin the analysis considering separately the equilibria of the two vector fields involved by assuming the constraint $y>0$.

\subsection{Equilibria of $\mathrm{F}^{ \pm}(\mathrm{x})$}

The vector field $\mathbf{F}^{-}(\mathbf{x})$ has only an equilibrium point at $\overline{\mathbf{x}}^{-}=\left(\frac{1}{b}, \sqrt{\frac{-d}{a}}, \frac{1}{b}\right)$ when $d<0$. It is a stable node since the eigenvalues are $\{-2 a,-b,-w\}$. Moreover, this is a real equilibrium point $\left(h\left(\overline{\mathbf{x}}^{-}\right)<0\right)$ for $d_{B E B^{-}}<d<0$, a virtual equilibrium $\left(h\left(\overline{\mathbf{x}}^{-}\right)>0\right)$ for $d<d_{B E B^{-}}$and is a boundary equilibrium point $\left(h\left(\overline{\mathbf{x}}^{-}\right)=0\right)$ for $d=d_{B E B^{-}}$which is the critical value for the BEB given by

$$
d_{B E B^{-}}=-a y_{r}^{2} \text {. }
$$

The equilibrium points of $\mathbf{F}^{+}(\mathbf{x})$ must fulfill the conditions

$$
x=\frac{1-y}{b}=z
$$

where $y$ must satisfy the quadratic equation

$$
(1+a b) y^{2}-y+b d=0 .
$$

Thus, the vector field $\mathbf{F}^{+}(\mathbf{x})$ have two equilibria at $\overline{\mathbf{x}}_{1}^{+}=\left(\bar{x}_{-}, \bar{y}_{-}, \bar{x}_{-}\right)$and $\overline{\mathbf{x}}_{2}^{+}=\left(\bar{x}_{+}, \bar{y}_{+}, \bar{x}_{+}\right)$if $d \leq d_{S N} ;$ where

$$
\bar{x}_{ \pm}=\frac{2(a+d)}{1+2 a b \pm \sqrt{1-d / d_{S N}}}, \bar{y}_{ \pm}=\frac{1 \pm \sqrt{1-d / d_{S N}}}{2(1+a b)},
$$

with

$$
d_{S N}=\frac{1}{4 b(1+a b)} .
$$

Note that, both equilibria are virtual for $0<d<d_{S N}$ because $\bar{y}_{ \pm}<1$ and $y_{r}>1$ implies $h<0$.

Furthermore, $\overline{\mathbf{x}}_{1}^{+}$is not feasible when $d \leq 0$ since that $\bar{y}_{-} \leq 0$. The stability of these equilibria can be analyzed considering only the dynamics with respect of the $(x, y)$ variables, since the dynamics on $z$ is stable (eigenvalue $\lambda_{3}=-w<0$ ). In this way, we only need to consider the reduced linearization matrix for $\overline{\mathbf{x}}_{2}^{+}$

$$
A_{+}=\left[\begin{array}{cc}
-b & -1 \\
1 & -a+\frac{d}{\bar{y}_{+}^{2}}
\end{array}\right],
$$


whose determinant and trace are given by

$$
\begin{gathered}
\operatorname{Det}\left(A_{+}\right)=1+a b-\frac{b d}{\bar{y}_{+}^{2}}, \\
\operatorname{Tr}\left(A_{+}\right)=-a-b+\frac{d}{\bar{y}_{+}^{2}} .
\end{gathered}
$$

The only relevant equilibrium point $\overline{\mathbf{x}}_{2}^{+}$remains virtual for small negatives values of $d$ and passes from virtual to real when $\bar{y}_{+}=y_{r}$, what happens for a certain value

$$
d_{B E B^{+}}=\frac{y_{r}\left(1-y_{r}\right)}{b}-a y_{r}^{2}=y_{r} \frac{1-(1+a b) y_{r}}{b}<0,
$$

where we have a BEB.

Thus, for $d<d_{B E B^{+}}$, we have $h\left(\overline{\mathbf{x}}_{2}^{+}\right)>0$, a situation not desirable since then $\operatorname{Det}\left(A_{+}\right)>0$ and $\operatorname{Tr}\left(A_{+}\right)<0$ and we could have a real stable equilibrium point for which $\left(\bar{y} \neq y_{r}\right)$. Therefore, the condition $d>d_{B E B^{+}}$is a constraint to be fulfilled by our control strategy.

\section{2. $\quad$ Sliding Mode Dynamics: pseudo-equilibria}

We recall that our aim is to design a SMC strategy leading to a stable pseudo-equilibrium point to be located in $\Sigma_{a s}$. Restricting the dynamics to $\Sigma_{a s}$, the sliding vector field as calculated from (13) and (14) turns out to be

$$
\mathbf{F}_{s}(\mathbf{x})=\frac{1}{k y-x}\left[\begin{array}{c}
b x^{2}-x+a y^{2}+d+k w y(x-z) \\
-k\left(b x^{2}-x+a y^{2}+d\right)-k w x(x-z) \\
w(k y-x)(x-z)
\end{array}\right],
$$

provided that $k y-x \neq 0$. The equilibria of $\mathbf{F}_{s}$ (i.e, the pseudo-equilibrium points of system (8), from Definition 3 , are $\widetilde{\mathbf{x}}_{ \pm}=\left(\widetilde{x}_{ \pm}, y_{r}, \widetilde{x}_{ \pm}\right)$, with

$$
\widetilde{x}_{ \pm}=\frac{1 \pm \gamma}{2 b}
$$

and

$$
\widetilde{\lambda}_{ \pm}=\frac{1 \mp \gamma}{2 y_{r}}
$$

where $\gamma \geq 0$ is such that $\gamma^{2}=1-4 b\left(d+a y_{r}^{2}\right)$ provided that this expression is positive. Thus, the points $\widetilde{\mathbf{x}}_{ \pm}$only exist for $\widetilde{x}_{ \pm} \neq k y_{r}$ and $d \leq d_{S N}^{s}$ with

$$
d_{S N}^{s}=\frac{1-4 a b y_{r}^{2}}{4 b}
$$

also note that (see Remark 3)

$$
d=d_{B E B^{+}} \Rightarrow \widetilde{\lambda}_{-}=\frac{1+\gamma}{2 y_{r}}=1,
$$

and so the points $\widetilde{\mathbf{x}}_{-}$and $\overline{\mathbf{x}}_{2}^{+}$collide, while

$$
d=d_{B E B^{-}} \Rightarrow \widetilde{\lambda}_{+}=\frac{1-\gamma}{2 y_{r}}=0,
$$

so that the points $\widetilde{\mathbf{x}}_{+}$and $\overline{\mathbf{x}}_{-}$also collide.

From the two possible pseudo-equilibrium points, we are interested in the operating point with

$$
\widetilde{x}_{-}=\frac{1-\gamma}{2 b}=\frac{2\left(d+a y_{r}^{2}\right)}{1+\gamma} .
$$


For the stability of such point, a first condition is that $\widetilde{\mathbf{x}}_{-}$belong to the attractive sliding region $\Sigma_{a s}$. The analysis is easier if we project both $\Sigma$ and $\mathbf{F}_{s}(\mathbf{x})$ on the $(x, y)$-plane, reducing so the dimension of the problem by simply substituting $z=z_{\Sigma}=\frac{y-y_{r}+k x}{k}$. Thus, the projection of $\Sigma_{a s}$ is given by

$$
\begin{aligned}
& L_{\mathbf{F}^{-}} h(\mathbf{x})=-b k x+(w-a) y-\frac{d}{y}+k-w y_{r}>0 \\
& L_{\mathbf{F}^{+}} h(\mathbf{x})=L_{\mathbf{F}^{-}} h(\mathbf{x})+x-k y<0
\end{aligned}
$$

where we already have done the above substitution.

From (30) and (31), all the points belonging to $\Sigma_{a s}$ fulfill the condition $k y-x>0$, and so we can desingularize the sliding vector field by considering the vector field $\mathbf{F}_{d s}(\mathbf{x})=(k y-x) \mathbf{F}_{s}(\mathbf{x})$. Moreover, the dynamics on the sliding mode can be analyzed in the $x y$-plane, substituting again $z=z_{\Sigma}$ and considering only the two first components of the desingularized sliding vector field $\mathbf{F}_{d s}$. Therefore, the differential equations that describe the dynamics on the sliding mode, projected on the $x y$-plane, can be expressed as

$$
\begin{aligned}
& \dot{x}=b x^{2}-x+a y^{2}+d-w y\left(y-y_{r}\right) \\
& \dot{y}=-k\left(b x^{2}-x+a y^{2}+d\right)+w x\left(y-y_{r}\right),
\end{aligned}
$$

for $k y-x>0, y>0$ and $x \in \mathbb{R}$.

The relevant equilibrium points for this reduced dynamical system are those with $y=y_{r}$, namely, the solutions of the quadratic equation

$$
b x^{2}-x+a y_{r}^{2}+d=0
$$

which are of course the previous values $\widetilde{x}_{ \pm}$given in (25). The jacobian matrix of the system (32)-(33) linearized around the equilibria $\widetilde{\mathbf{x}}_{ \pm}$is expressed as

$$
J_{ \pm}=\left[\begin{array}{cc}
-1+2 b \widetilde{x}_{ \pm} & y_{r}(2 a-w) \\
k\left(1-2 b \widetilde{x}_{ \pm}\right) & w \widetilde{x}_{ \pm}-2 a y_{r} k
\end{array}\right]
$$

whose determinant is

$$
\operatorname{Det}\left(J_{ \pm}\right)=w\left(1-2 b \widetilde{x}_{ \pm}\right)\left(k y_{r}-\widetilde{x}_{ \pm}\right)
$$

and its trace

$$
\operatorname{Tr}\left(J_{ \pm}\right)=(w+2 b) \widetilde{x}_{ \pm}-2 a k y_{r}-1 .
$$

Assuming $\widetilde{\mathbf{x}}_{-} \in \Sigma_{a s}$, we have that $\operatorname{Det}\left(J_{-}\right)>0$, because $\widetilde{x}_{-}<1 / 2 b$ and $\widetilde{x}_{-}<k y_{r}$. Thus, $\widetilde{\mathbf{x}}_{-}$is stable (node or focus) in $\Sigma_{a s}$ whenever $\operatorname{Tr}\left(J_{-}\right)<0$, that is, for

$$
k>\frac{(w+2 b) \widetilde{x}_{-}-1}{2 a y_{r}} .
$$

Regarding $\widetilde{\mathbf{x}}_{+}$, we have $\operatorname{Det}\left(J_{+}\right)<0$ since $\widetilde{x}_{+}>1 / 2 b$. Therefore, it is a pseudo-saddle in the region $\Sigma_{a s}$. Clearly, the pseudo-equilibrium point $\widetilde{\mathbf{x}}_{-}$must be chosen as the operating point for the feedback control system.

\subsection{Boundary Equilibrium Bifurcations (BEB)}

Let us write explicitly the dependence on the parameter $d$ of the positions of the equilibria $\overline{\mathbf{x}}^{-}(d)$ of vector field $\mathbf{F}^{-}, \overline{\mathbf{x}}_{1,2}^{+}(d)$ of vector field $\mathbf{F}^{+}$, the pseudo-equilibria $\widetilde{\mathbf{x}}_{ \pm}(d)$ and the values $\widetilde{\lambda}_{\mp}(d)$. In order to detect the occurrence of a BEB, let us consider first the analysis of the vector field $\mathbf{F}^{-}$and then for the vector field $\mathbf{F}^{+}$.

The equilibrium point $\overline{\mathbf{x}}^{-}(d)$ of the vector field $\mathbf{F}^{-}$that describes a smooth curve in $\mathbb{R}^{3}$ defined for $d<0$, crossing transversally the switching boundary $\Sigma$ at the point $\overline{\mathbf{x}}^{-}\left(d_{B E B^{-}}\right)=\left(1 / b, y_{r}, 1 / b\right)$ for the critical value $d=d_{B E B^{-}}$, because $h\left(\overline{\mathbf{x}}^{-}\left(d_{B E B^{-}}\right)\right)=0$ and

$$
h^{\prime}\left(\overline{\mathbf{x}}^{-}\left(d_{B E B^{-}}\right)\right)=-\frac{1}{2 a y_{r}}<0 .
$$


The value of the negative derivative with respect to $d$ in (38) indicates that $\overline{\mathbf{x}}^{-}(d)$ is a virtual equilibrium point for $d<d_{B E B^{-}}$and real for $d>d_{B E B^{-}}$. At the critical value $d=d_{B E B^{-}}$, the equilibrium $\overline{\mathbf{x}}^{-}(d)$ collides with the pseudo-equilibrium point $\widetilde{\mathbf{x}}_{+}(d)$, giving rise to a boundary equilibrium point. This is a point on the border line between the attractive/repulsive sliding regions and the crossing region, since $\widetilde{\lambda}_{+}\left(d_{B E B^{-}}\right)=0$. The pseudo-equilibrium point $\widetilde{\mathbf{x}}_{+}(d)$ crosses this border line transversally, because

$$
\widetilde{\lambda}_{+}^{\prime}\left(d_{B E B^{-}}\right)=\frac{b}{y_{r}}>0
$$

The positive derivative in (39) indicates that $\widetilde{\mathbf{x}}_{+}(d)$ is a virtual pseudo-equilibrium for $d<d_{B E B^{-}}$and real for $d>d_{B E B^{-}}$. Thus, when $d=d_{B E B^{-}}$a BEB of type non-smooth fold occurs [di Bernardo et al., 2008a,b; Pagano et al., 2011].

For the vector field $\mathbf{F}^{+}$the equilibrium $\overline{\mathbf{x}}_{1}^{+}(d)$ is always virtual because $y_{r}>1$. The other equilibrium point, $\overline{\mathbf{x}}_{2}^{+}(d)$, describes a smooth curve in $\mathbb{R}^{3}$ that crosses transversally the switching boundary $\Sigma$ at the point $\overline{\mathbf{x}}_{2}^{+}\left(d_{B E B^{+}}\right)=\left(\frac{1-y_{r}}{b}, y_{r}, \frac{1-y_{r}}{b}\right)$ for the critical value $d=d_{B E B^{+}}$, because $h\left(\overline{\mathbf{x}}_{2}^{+}\left(d_{B E B^{+}}\right)\right)=0$ and

$$
h^{\prime}\left(\overline{\mathbf{x}}_{2}^{+}\left(d_{B E B^{+}}\right)\right)=-\frac{b}{\left|2 y_{r}(1+a b)-1\right|}<0 .
$$

The negative derivative respect to $d$ in (40) states that $\overline{\mathbf{x}}_{2}^{+}(d)$ is a real equilibrium point for $d<d_{B E B^{+}}$ and virtual for $d>d_{B E B^{+}}$. At the critical value $d=d_{B E B^{+}}, \overline{\mathbf{x}}_{2}^{+}(d)$ collides with the pseudo-equilibrium point $\widetilde{\mathbf{x}}_{-}(d)$ establishing a boundary equilibrium point. This is a point over the border line between the attractive/repulsive sliding regions and the crossing region, since $\widetilde{\lambda}_{-}\left(d_{B E B^{+}}\right)=1$. The pseudo-equilibrium point $\widetilde{\mathbf{x}}_{-}(d)$ crosses this border line transversally since

$$
\tilde{\lambda}_{-}^{\prime}\left(d_{B E B^{+}}\right)=-\frac{b}{y_{r}\left|2 y_{r}-1\right|}<0 .
$$

The negative derivative in (41) shows that $\widetilde{\mathbf{x}}_{-}(d)$ is a virtual pseudo-equilibrium point for $d<d_{B E B^{+}}$and real for $d>d_{B E B^{+}}$. Thus, at the critical value $d=d_{B E B^{+}}$occurs a BEB classified as of persistence type [di Bernardo et al., 2008a,b; Pagano et al., 2011].

Simulation results of the DPWS dynamical system (8) are shown in Fig. 8, considering $b=0.06$, $w=0.6, y_{r}=1.2, k=1, a=0.3$ and $d \in\{-5,-4.432,-4\}$. Moreover, it is possible to appreciate in this figure the occurrence of a BEB of persistence type where the stable real pseudo-node $\left(\widetilde{\mathbf{x}}_{-}\right)$becomes virtual and the virtual stable node $\left(\overline{\mathbf{x}}_{2}^{+}\right)$of the vector field $\mathbf{F}^{+}$becomes real.

\subsection{Hopf Bifurcation in the sliding dynamics}

The Hopf bifurcation analysis will be made only for the pseudo-equilibrium $\widetilde{\mathbf{x}}_{-}$that is the point of interest from the control viewpoint. Recalling (37), dynamical system (32)-(33), linearized around the pseudoequilibrium point in the $(x, y)$-plane corresponding to $\widetilde{\mathbf{x}}_{-}$, has purely imaginary eigenvalues when $k=k_{\mathrm{Hopf}}$ with

$$
k_{\mathrm{Hopf}}=\frac{(w+2 b) \widetilde{x}_{-}-1}{2 a y_{r}}=\frac{w-(2 b+w) \gamma}{4 a b y_{r}},
$$

provided that the determinant (35) is positive, i.e.,

$$
k_{\text {Hopf }}>\frac{\widetilde{x}_{-}}{y_{r}}=\frac{1-\gamma}{2 b y_{r}} .
$$

It can be shown that there exists a subcritical Hopf bifurcation (SubHB) for $k=k_{\text {Hopf }}$, leading for $k>k_{\text {Hopf }}$ to an unstable limit cycle surrounding the stable pseudo-equilibrium $\widetilde{\mathbf{x}}_{-}$.

Choosing $d=1.5, a=0.06$ and $k$ as the bifurcation parameter, a Hopf bifurcation (HB) is detected, as shown in Fig. 9, where the straight line denotes the position of the pseudo-equilibrium point $\widetilde{\mathbf{x}}_{-}$in the $x$-axis. The red part of this line corresponds to an unstable pseudo-equilibrium point and the blue part to a stable pseudo-equilibrium point. The green curve represents the position of the two-fold point in the $x$-axis, 


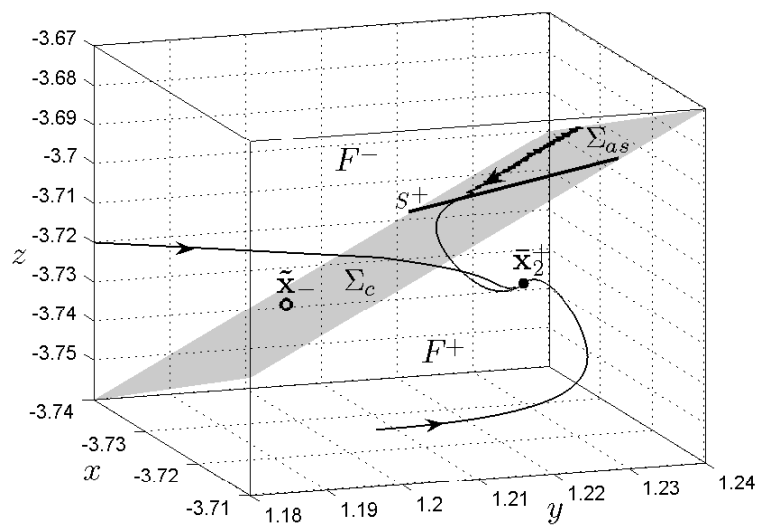

(a)Case $d=-5<d_{B E B^{+}}: \overline{\mathbf{x}}_{2}^{+}$real and $\widetilde{\mathbf{x}}_{-}$virtual.

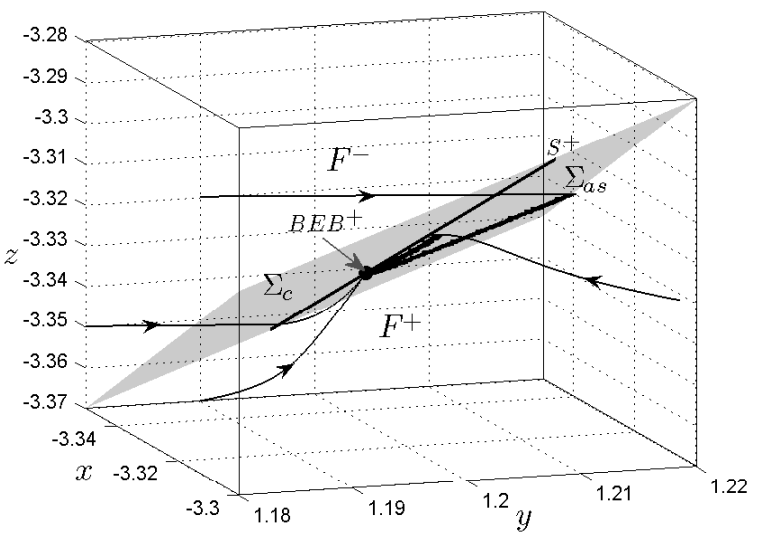

(b) $\mathrm{BEB}^{+}$bifurcation when $d=d_{B E B^{+}}=-4.432$ where $\overline{\mathbf{x}}_{2}^{+}\left(d_{B E B^{+}}\right)=\widetilde{\mathbf{x}}_{-}\left(d_{B E B^{+}}\right)$is a boundary equilibrium point.

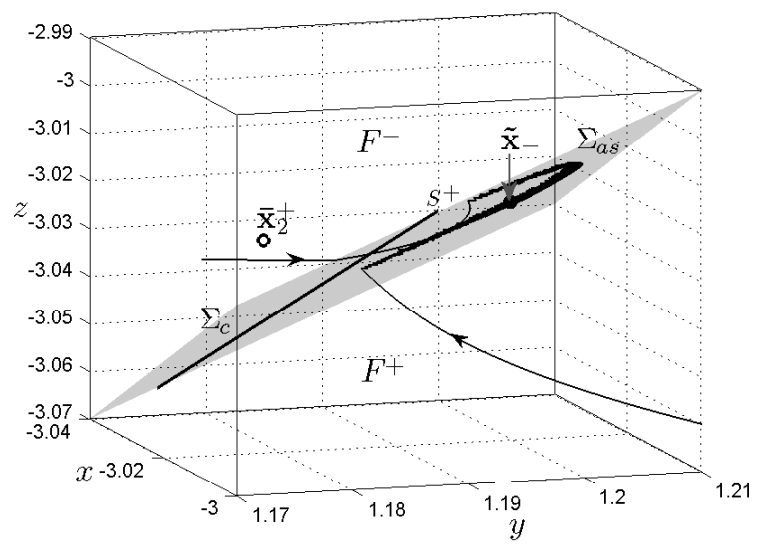

(c)Case $d=-4>d_{B E B^{+}}: \overline{\mathbf{x}}_{2}^{+}$virtual and $\widetilde{\mathbf{x}}_{-}$real.

Figure 8. State-space diagrams of system (8) for persistence BEB case.

i.e., $\widehat{x}_{-}=k \widehat{y}_{-}$with $\widehat{y}_{-}$given in (43). The dotted curve denotes the amplitude of the limit cycles, that in this case are unstable due to a SubHB. The dashed vertical line represents the homoclinic connection (HC) bifurcation.

The HB and the HC bifurcation here occur differently from what is known in the literature on dynamical systems. In this case the limit cycle confined to the sliding region $\Sigma_{a s}$, originated by the HB disappears when it touches the two-fold point, that is the limit of $\Sigma_{a s}$. The limit cycle disappears at an HC that is non smooth at the two-fold point. This point is a saddle equilibrium point of the desingularized sliding vector field $\mathbf{F}_{d s}$, but it is not for the sliding vector field $\mathbf{F}_{s}$. However, the dynamics of saddle type on $\Sigma_{a s}$, near this point, is present (see Figures 10 and 11).

Changes on the parameter $k$ do not affect the position of the pseudo-equilibrium point, just only its stability, thus for $0<k<k_{\text {Hopf }}=1.754$ the pseudo-equilibrium point $\widetilde{\mathbf{x}}_{-}$is unstable and for $k>k_{\mathrm{Hopf}}=$ 1.754 is stable. The SubHB occurs at $k=k_{\mathrm{Hopf}}=1.754$ and the unstable limit cycle (uLC) exists for

$$
1.754<k<2.174 \text {. }
$$

The state-space diagram of system (8) for $k=2$, has an uLC around a stable pseudo-focus, as shown in Fig. 10. This limit cycle disappears in a $\mathrm{HC}$ when touches a two-fold point for $k=2.174$, as depicted in Fig. 11. 


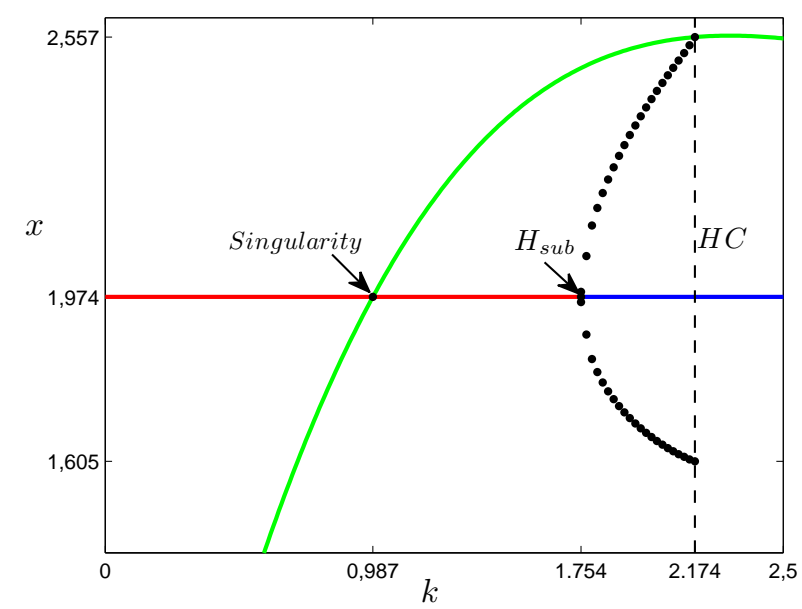

Figure 9. Bifurcation diagram in $(k, \widetilde{x})$-plane for $b=0.06, w=0.6, y_{r}=2, d=1.5$ and $a=0.06$

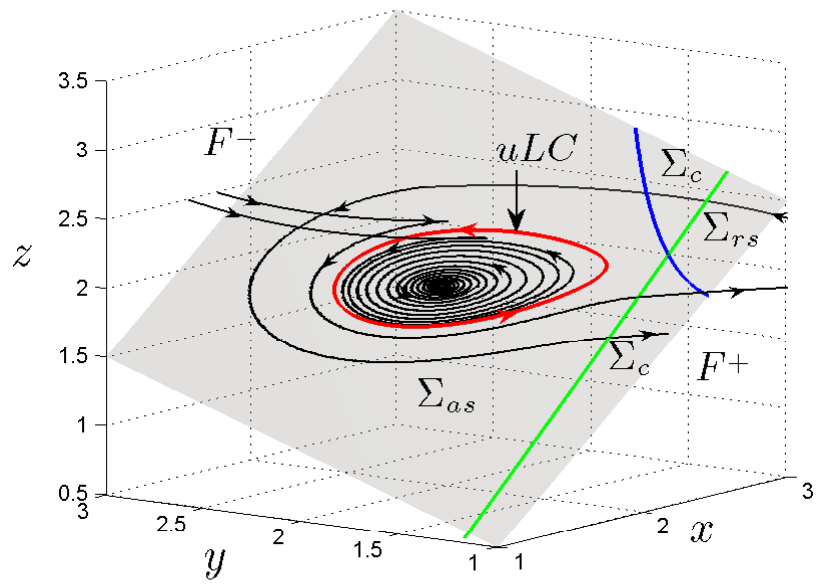

Figure 10. State-space diagrams of system (8). Green line $L_{\mathbf{F}^{-}} h(\mathbf{x})=0$. Blue line $L_{\mathbf{F}^{+}} h(\mathbf{x})=0$. Parameters $b=0.06$, $w=0.8, y_{r}=2, d=1.5, a=0.06$ and $k=2$.

\subsection{T-Singularity Bifurcation}

The dynamical system given by (8) can have up to two points of two-fold type denoted by $\widehat{\mathbf{x}}_{ \pm}$as shown in Fig. 12. The TS bifurcation can occur in two different ways, one involving the pseudo-equilibrium point $\widetilde{\mathbf{x}}_{-}$and another involving the pseudo-equilibrium $\widetilde{\mathbf{x}}_{+}$. Here, we will analyze only the bifurcation involving $\widetilde{\mathbf{x}}_{-}$since it is a stable equilibrium point (desired operating point).

Using Definition 1 and equations (30)-(31), it is possible to determine the two-fold points. These points whenever exist have the form $\widehat{\mathbf{x}}_{ \pm}=\left(k \widehat{y}_{ \pm}, \widehat{y}_{ \pm}, \widehat{z}_{ \pm}\right)$, with $\widehat{z}_{ \pm}=\frac{\left(1+k^{2}\right) \widehat{y}_{ \pm}-y_{r}}{k}$ and

$$
\widehat{y}_{ \pm}=\frac{k-w y_{r} \pm \sqrt{\left(k-w y_{r}\right)^{2}-4 d\left(a+b k^{2}-w\right)}}{2\left(a+b k^{2}-w\right)},
$$

provided that $\left(a+b k^{2}-w\right) \neq 0$ and $\left(k-w y_{r}\right)^{2}-4 d\left(a+b k^{2}-w\right)>0$. The collision of the pseudo-equilibrium $\widetilde{\mathbf{x}}_{-}$with the two-fold $\widehat{\mathbf{x}}_{+}$(or $\left.\widehat{\mathbf{x}}_{-}\right)$happens at the point $\widehat{\mathbf{x}}=\left(k y_{r}, y_{r}, k y_{r}\right)$ when

$$
\widetilde{x}_{-}=k y_{r} .
$$




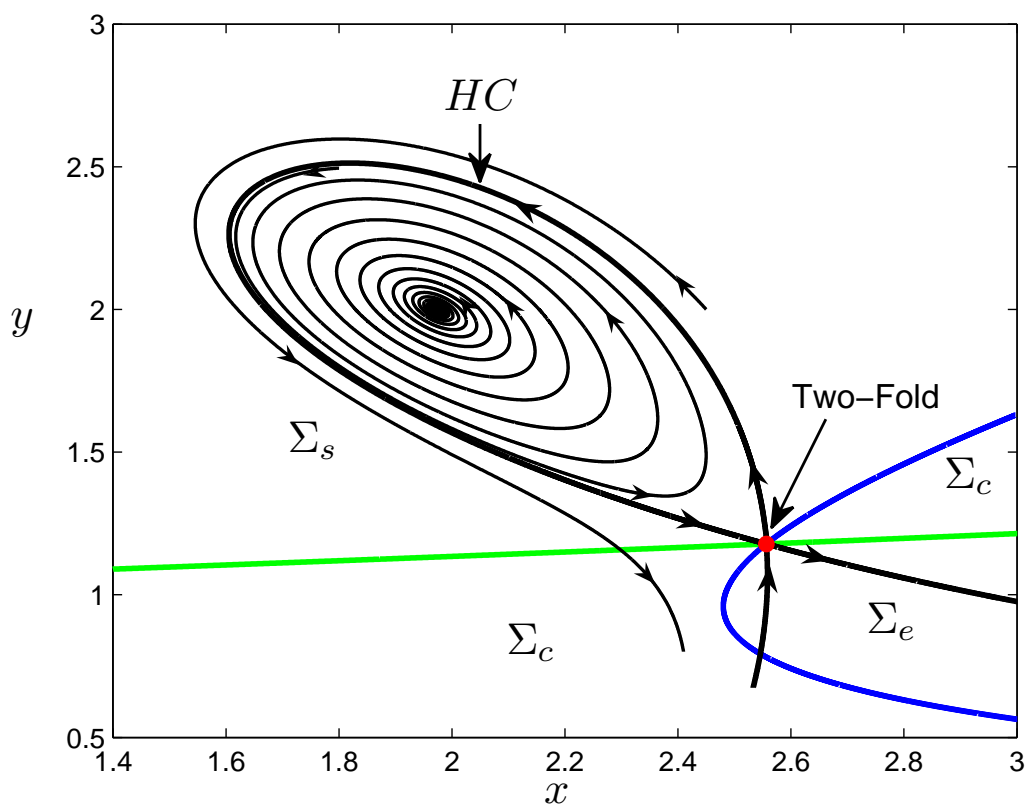

Figure 11. Bifurcation diagram showing a HC bifurcation with parameters $b=0.06, w=0.6, y_{r}=2, d=1.5, a=0.06$ and $k=2.174$.

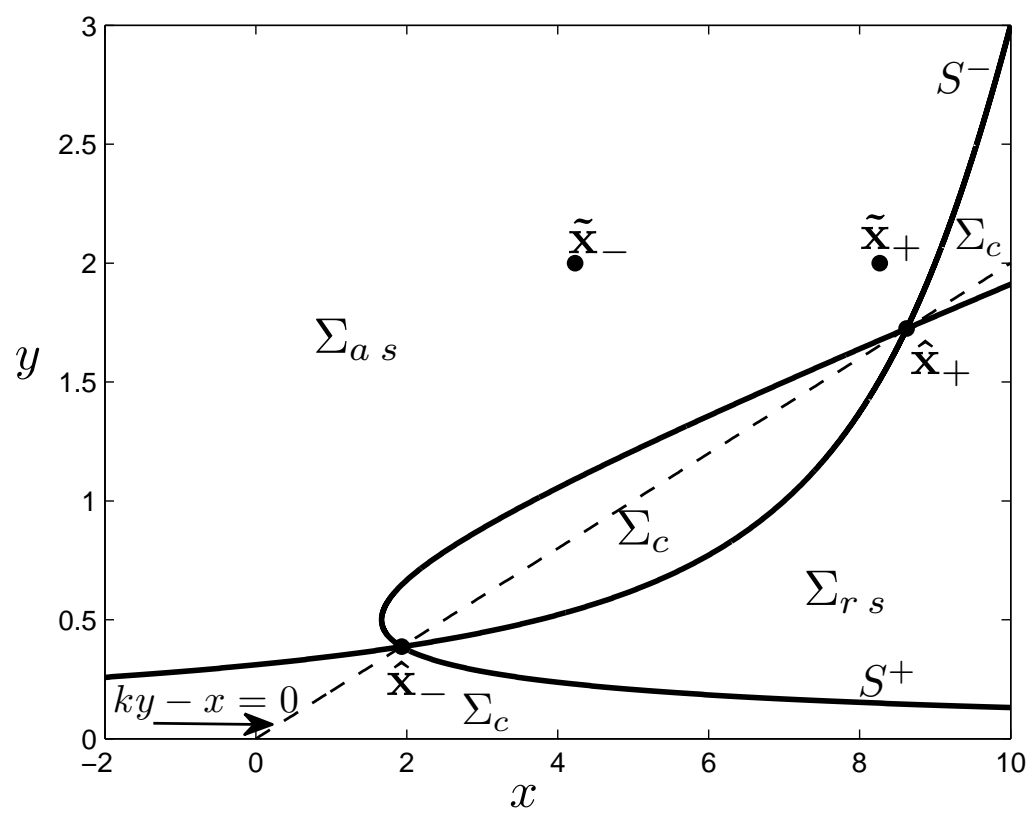

Figure 12. View of $\Sigma$ projected in the $x y$-plane for parameters $b=0.08, w=0.6, y_{r}=2, d=1.2, a=0.4$ and $k=5$.

Now, considering parameter $d$ as a bifurcation parameter, equation (44) can be true when $d=d_{T S}$, where

$$
d_{T S}=-a y_{r}^{2}+k y_{r}\left(1-b y_{r} k\right)
$$


Then $\gamma=\left|2 b k y_{r}-1\right|$ so that (44) is definitely true if the control parameter $k$ satisfies the condition

$$
k<\frac{1}{2 b y_{r}} .
$$

In this case, only for $d_{B E B^{+}}<d<d_{T S}$, the point $\widetilde{\mathbf{x}}_{-} \in \Sigma_{a s}$, while, for $d_{T S}<d<d_{S N}^{s} \widetilde{\mathbf{x}}_{-} \in \Sigma_{r s}$. Note that under condition (46) it is possible the collision of the pseudo-equilibrium point $\widetilde{\mathbf{x}}_{-}$with the two-fold point $\widehat{\mathbf{x}}$ at the bifurcation condition $d=d_{T S}$ since $d_{B E B^{+}}<d_{T S}<d_{S N}^{S}$.

Based on Definition 1 and in the following equations

$$
\begin{aligned}
& L_{\mathbf{F}^{+}}^{2} h(\widehat{\mathbf{x}})=\frac{1}{y_{r}}\left(1-(1+b k) y_{r}\right)\left(\left(1+k^{2}\right) y_{r}+k\left(b y_{r} k^{2}-k-(b+w) y_{r}+2 y_{r} a\right)\right) \\
& L_{\mathbf{F}^{-}}^{2} h(\widehat{\mathbf{x}})=\frac{k}{y_{r}}\left(1-b y_{r} k\right)\left(b y_{r} k^{2}-k-(w+b) y_{r}+2 y_{r} a\right)
\end{aligned}
$$

with $k$ fulfilling (46), it can be proved that $L_{\mathbf{F}^{-}}^{2} h(\widehat{\mathbf{x}})>0$ and $L_{\mathbf{F}^{+}}^{2} h(\widehat{\mathbf{x}})<0$, provided that the load parameter $a$ meets the inequality

$$
a>\frac{1}{2 y_{r}}\left(-b y_{r} k^{2}+k+(b+w) y_{r}\right) .
$$

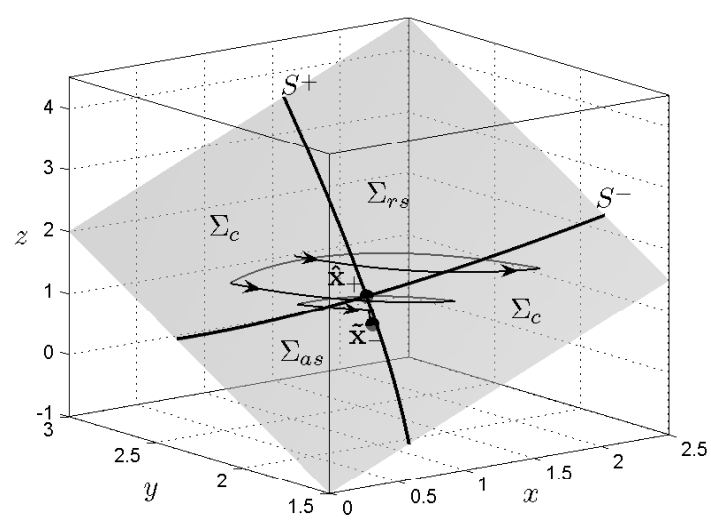

(a) $d<d_{T S}$

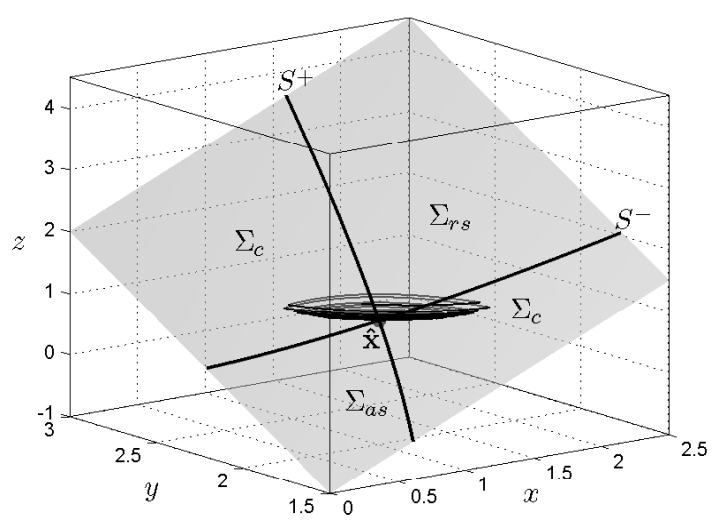

(b) $d=d_{T S}$

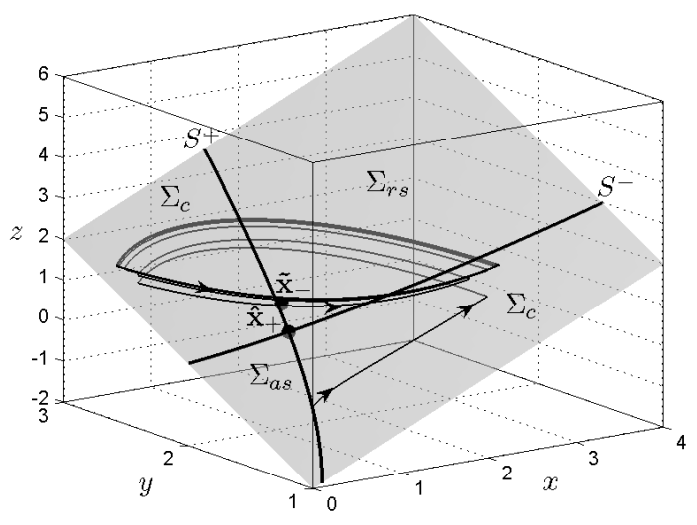

(c) $d>d_{T S}$

Figure 13. State-space diagrams of system (8) considering $w=0.6, b=0.06, y_{r}=2, a=0.5$ and $k=0.5$. (a) $d=-1.1$. (b) $d=d_{T S}=-1.06$. (c) $d=-1$. 
In order to analyze the stability of the pseudo-equilibrium point $\widetilde{\mathbf{x}}_{-}$, near the TS, i.e. for $d \approx d_{T S}$, $(k, a)$ fulfilling (46)-(47), equations (35)-(36) will be considered. The critical value $d=d_{T S}$ implies that $\widetilde{x}_{-}=k y_{r}$, thus $\operatorname{Det}\left(J_{-}\right)=0$ and $\operatorname{Tr}\left(J_{-}\right)=(w+2 b-2 a) k y_{r}-1<0$ for all $k$ and $a$ fulfilling (46) and (47), respectively, see Fig. 13(b) where $\widehat{\mathbf{x}}=\widetilde{\mathbf{x}}_{-}\left(d_{T S}\right)=\widehat{\mathbf{x}}_{+}\left(d_{T S}\right)$ is a TS point. For $d<d_{T S}$, we have that $\operatorname{Det}\left(J_{-}\right)>0$, but sufficiently close to zero when $d$ is sufficiently close to $d_{T S}$. Therefore, $\widetilde{\mathbf{x}}_{-} \in \Sigma_{a s}$ is a stable pseudo-node when it is sufficiently close to the TS (see Fig. 13(a)). Now, for $d>d_{T S}$ we have $\operatorname{Det}\left(J_{-}\right)<0$, and hence $\widetilde{\mathbf{x}}_{-} \in \Sigma_{r s}$ is a pseudo-saddle point.

In this way, a TS bifurcation occurs when $d=d_{T S}$, given in (45), for all $(k, a)$ fullfilling (46) and (47). This bifurcation gives rise to a stable CLC, born at the TS (see Fig. 13(b)), crossing the $\Sigma_{c}$ regions (see Fig. 13(c)) for $d>d_{T S}$. Simulation results corresponding to system (8) are shown in Fig. 13 where it is possible to visualize the TS bifurcation and the birth of a stable CLC.

As already mentioned $\operatorname{Det}\left(J_{-}\right)=0$ when $d=d_{T S}$. In fact, for the desingularized sliding vector field $\mathbf{F}_{d s}$ in $x y$-plane, the points $\widehat{\mathbf{x}}_{ \pm}$are also equilibria. Thus, when $d=d_{T S}$, with (46) and (47) true, the equilibrium points $\widehat{\mathbf{x}}_{+}$and $\widetilde{\mathbf{x}}_{-}$of $\mathbf{F}_{d s}$, change mutually their stability. In short, the TS bifurcation is seen as a Transcritical bifurcation for the desingularized sliding dynamics.

\subsection{Saddle-Node Bifurcation in the sliding dynamics}

Recall that the pseudo-equilibria $\widetilde{\mathbf{x}}_{ \pm}$are being defined for $d \leq d_{S N}^{s}$, as given by (25) and (27). For $d=d_{S N}^{s}$ they collide, i.e., there exists only one equilibrium point in the sliding field and the determinant in (35) goes to zero because $\widetilde{\mathbf{x}}_{ \pm}\left(d_{S N}^{s}\right)=\frac{1}{2 b}$.

The saddle-node bifurcation (SN) bifurcation in the sliding dynamics can be of two different types: (i) a $\mathrm{SN}^{r s}$ bifurcation occurs when $d=d_{S N}^{s}$ and $k<\frac{1}{2 b y_{r}}$, so that for $d<d_{S N}^{s}$ and near the critical value $d_{S N}^{s}$, $\widetilde{\mathbf{x}}_{-}$is a pseudo-saddle and $\widetilde{\mathbf{x}}_{+}$is a pseudo-node, both in $\Sigma_{r s}$; (ii) a $\mathrm{SN}^{a s}$ bifurcation occurs when $d=d_{S N}^{s}$ and $k>\frac{1}{2 b y_{r}}$, so that for $d<d_{S N}^{s}$, and near the critical value $d_{S N}^{s}, \widetilde{\mathbf{x}}_{-}$is a pseudo-node or pseudo-focus (stable or unstable), depending on the sign of the trace (36)), and $\widetilde{\mathbf{x}}_{+}$is a pseudo-saddle, both in $\Sigma_{a s}$. When $d=d_{S N}^{s}$ and $k=\frac{1}{2 b y_{r}}$ (see point $\mathrm{P}$ in Figs. 15 and 16), we have a degenerate case where the SN occurs on the two-fold point $\widehat{\mathbf{x}}_{-}$if $w>2 a$ or on $\widehat{\mathbf{x}}_{+}$if $w<2 a$; a more degenerate case appears when $w=2 a$, imply the collision of four singular points (the two points of pseudo-equilibria and the two points of double tangency), i.e., $\widetilde{\mathbf{x}}_{+}=\widetilde{\mathbf{x}}_{-}=\widehat{\mathbf{x}}_{+}=\widehat{\mathbf{x}}_{-}$.

Two bifurcation diagrams in $\left(d, \widetilde{x}_{ \pm}\right)$-plane are shown in Fig. 14, where the inferior branch of the parabolic curve represents $\widetilde{x}_{-}(d)$, and the superior branch represents $\widetilde{x}_{+}(d)$. The continuous part corresponds to the stable equilibrium manifold, and the dashed curve corresponds to the unstable equilibrium manifold. A SN of type $\mathrm{SN}^{r s}$ that occurs in the escaping region $\Sigma_{r s}$ is depicted in Fig. 14(a) and a SN of type $\mathrm{SN}^{a s}$ for the attractive sliding region $\Sigma_{a s}$ in Fig. 14(b).

\subsection{Summary of the bifurcation analysis}

In short, summarizing the bifurcation analysis, we can state the following result.

Proposition 1. Fixed $y_{r}>1, b>0, w>0, k>0$, and assuming as the main parameters $a>0$ and $d \in \mathbb{R}$ for system (8), where $h(\mathbf{x})$ is given by (6), the following statements hold.

(a) For $d>0$, the system has no real equilibrium points and only for $d \leq 0$ we have:

(a.1) a stable equilibrium point $\overline{\mathbf{x}}_{-}$(far from the desired operating point) if $d_{B E B^{-}} \leq d \leq 0$;

(a.2) a stable equilibrium point $\overline{\mathbf{x}}_{2}^{+}$if $d<d_{B E B^{+}}$, which comes from a BEB bifurcation of the desired operating point. Therefore, we must assure that $d>d_{B E B^{+}}$, what imposes a limitation on the negative values of power $P$. Otherwise, the equilibrium value of $y>0$ is not longer equal to $y_{r}$.

(b) To assure the existence of our desired operating point $\widetilde{\mathbf{x}}_{-}$belonging to $\Sigma_{a s}$, the following conditions must be fulfilled.

$$
\begin{aligned}
& \text { (b.1) } d_{B E B^{+}}(a)<d<d_{S N}^{s}(a) \text { when } k>\frac{1}{2 b y_{r}} \\
& (b .2) d_{B E B^{+}}(a)<d<d_{T S}(a) \text { when } k \leq \frac{1}{2 b y_{r}} .
\end{aligned}
$$




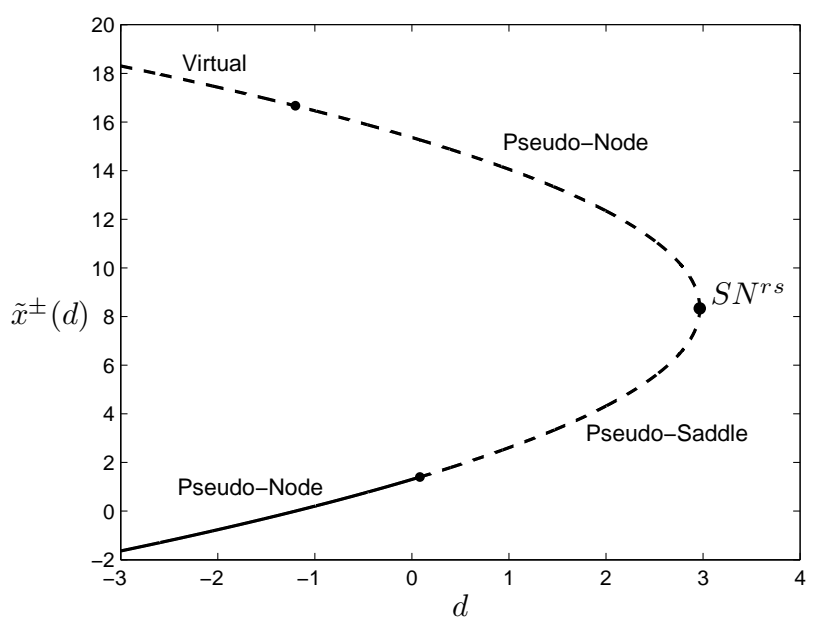

(a) $k=0.7<\frac{1}{2 b y_{r}}$.

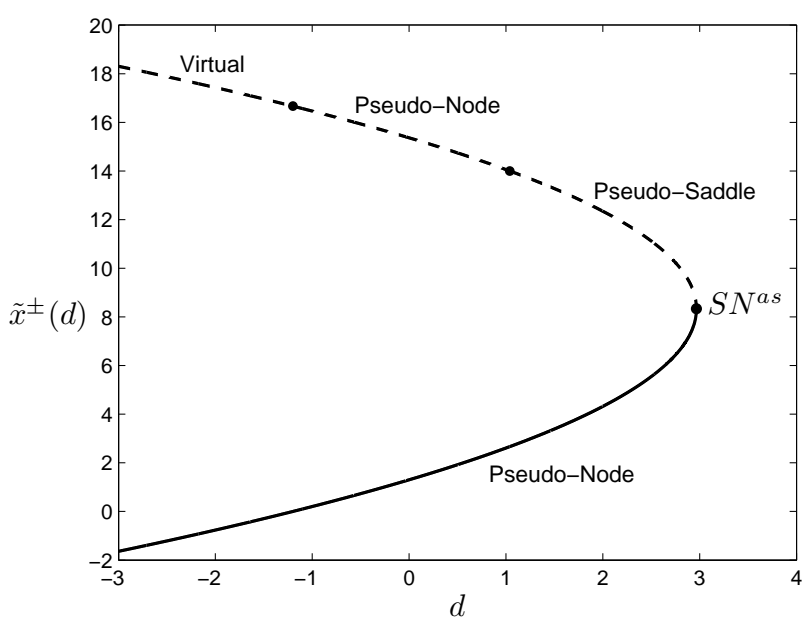

(b) $k=7>\frac{1}{2 b y_{r}}$.

Figure 14. Bifurcation diagrams showing a SN bifurcation with parameters $b=0.06, w=0.6, y_{r}=2$ and $a=0.3$.

Furthermore, under above conditions (b.1) and (b.2), the operating point will be stable whenever $k>k_{\text {Hopf }}$ given in (42).

Finally, we remark that another possible bifurcation to appear is the so-called Bogdanov-Takens (BT) bifurcation. This codimension-two bifurcation is characterized by $\operatorname{Det}\left(J_{-}\right)=\operatorname{Tr}\left(J_{-}\right)=0$ or $\operatorname{Det}\left(J_{+}\right)=$ $\operatorname{Tr}\left(J_{+}\right)=0$.

\section{Bifurcation set}

The above bifurcation analysis can be appropriately summarized in a bifurcation set (see Fig. 15) in terms of the load parameters $a, d$ and the control parameter $k$.

The bifurcation set in $\mathbb{R}^{3}$ for the parameters $a>0, k>0$ and $d \in \mathbb{R}$ is shown in Fig. 15. Points $(d, k, a)$ on the plane in gray-color (equation (27)) determine a SN bifurcation. Points $(d, k, a)$ on the surface defining by (42) in blue-color stand for a SubHB corresponding to the pseudo-equilibrium $\widetilde{\mathbf{x}}_{-}$. Points $(d, k, a)$ on the curves in cyan-color determine a BT bifurcation. Points $(d, k, a)$ on the plane in green-color (equation (17)) determine a BEB classified as a non-smooth fold corresponding to the pseudo-equilibrium $\widetilde{\mathbf{x}}_{+}$and the 
equilibrium $\overline{\mathbf{x}}^{-}$of vector field $\mathbf{F}^{-}$. Points $(d, k, a)$ on the surface defined by (45) for $k$ in (46) in red-color represent the collision of the pseudo-equilibrium $\widetilde{\mathbf{x}}_{-}$with a two-fold point. The region on this surface such that $a$ satisfies (47) determines the TS bifurcation.

We also show in Fig. 15 three sections of the bifurcation set: (i) $(k, a)$ for $d=1.5$, (ii) ( $d, a$ ) for $k=1.5$ and (iii) $(d, k)$ for $a=0.15$; allow us to show the stability regions and the bifurcation curves. In parameter region I, system (8) have no pseudo-equilibria. In parameter region II, the pseudo-equilibrium $\widetilde{\mathbf{x}}_{-}$is unstable and in parameter regions III and IV is stable. The pseudo-equilibrium $\widetilde{\mathbf{x}}_{+}$is always unstable, being virtual in region IV.

In the parameter region denoted by $u L C$, in the section $(k, a)$ with $d=1.5$, there exists an unstable limit cycle around of a stable pseudo-focus $\widetilde{\mathbf{x}}_{-}$. The curve denoted by $H C$ in black-color cooresponds to an homoclinic connection where the limit cycle disappears. This curve have been computed numerically and it is not shown in the other pictures.
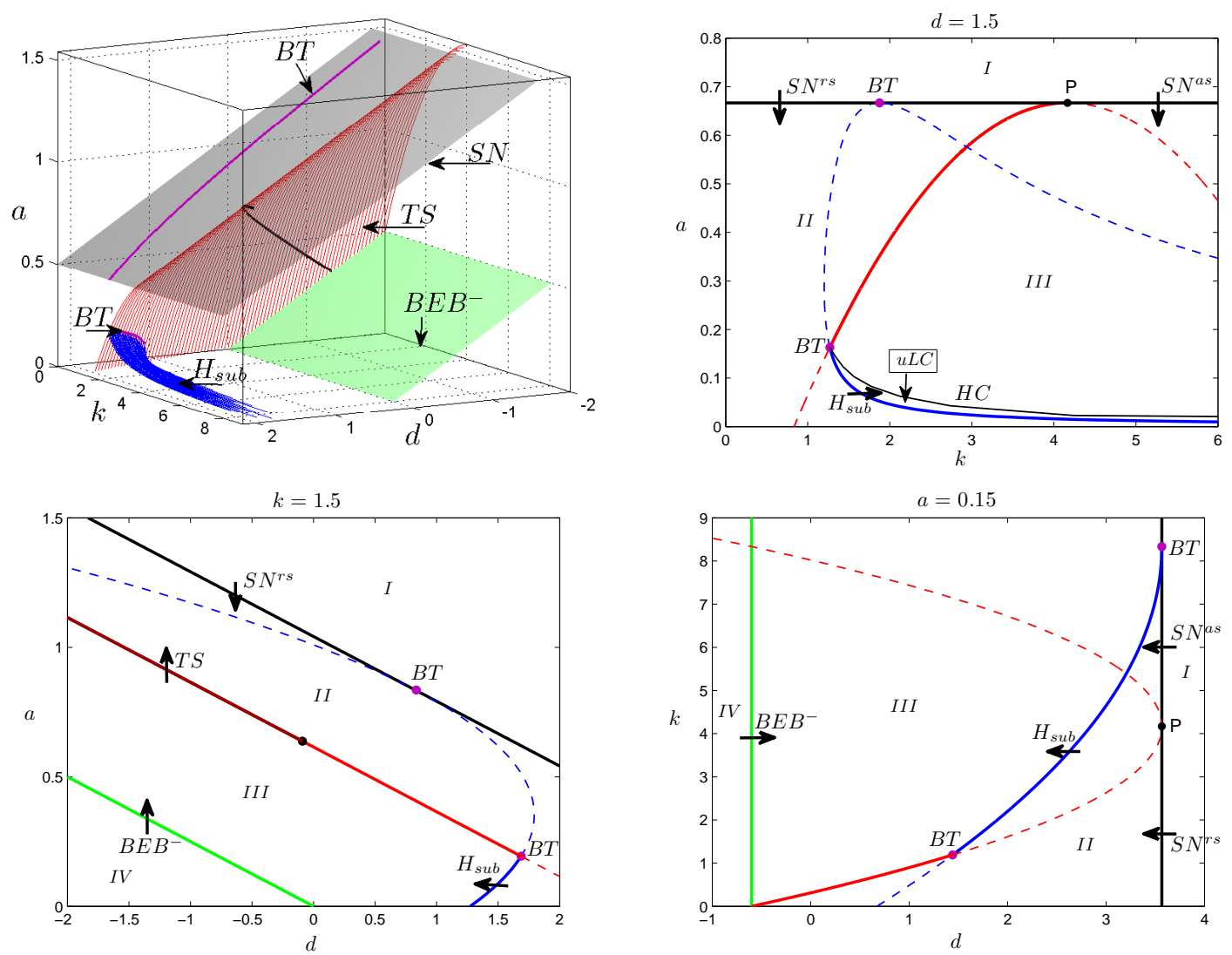

Figure 15. Bifurcation set in $(d, k, a)$-space with parameters $b=0.06, w=0.6$ and $y_{r}=2$.

In the parametric section $(d, a)$ with $k=1.5$, the brown-color line points out the TS bifurcation, where near the bifurcation we have two different situations: (i) for points $(d, a)$ into region II there exists a stable CLC crossing the region $\Sigma_{c}$ or (ii) for points $(d, a)$ into region III there exists an unstable CLC with a dynamics of saddle type crossing the region $\Sigma_{c}$. To prove the existence and the stability of these CLC it is necessary to use the Poincaré maps theory applied to DPWS systems, for the sake of brevity it is not shown here.

The vector field $\mathbf{F}^{+}$has a real stable equilibrium point only when $d<d_{B E B^{+}}$. This bifurcation is not shown in Fig. 15 since for these parameters values we have that $d_{B E B^{+}} \ll 0$. In Fig. 16 we assume that $b=0.2, w=0.8, y_{r}=1.2$ with $a=0.15$. In this case, in the region $\mathrm{V}$, the pseudo-equilibrium $\widetilde{\mathbf{x}}_{-}$is virtual and the dynamical system (8) has only one real and stable equilibrium point,i.e., the point $\overline{\mathbf{x}}_{2}^{+}$of the vector 
field $\mathbf{F}^{+}$. The different regions showed on the bifurcation set are delimited by solid lines.

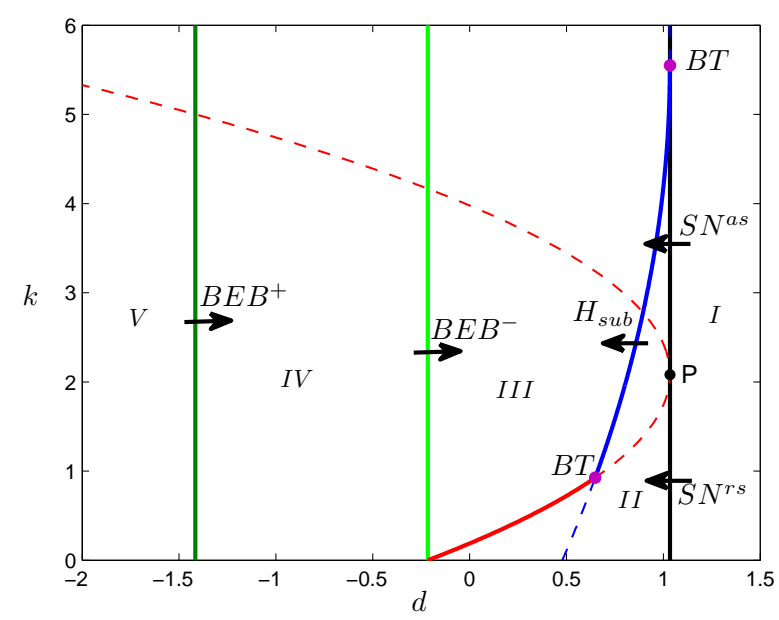

Figure 16. Bifurcation set in $(d, k)$-plane with parameters $b=0.2, w=0.8, y_{r}=1.2$ and $a=0.15$.

From the former analysis, we can give the following practical guidelines to choose the parameter $k$ to guarantee the robustness of our SMC design, regarding variations of parameters $a$ and $d$ :

(i) $k$ must be selected such that $k \in \mathbf{K}=\left\{k>\frac{\widetilde{x}_{-}}{y_{r}}=\frac{1-\gamma}{2 b y_{r}}\right\} \cap\left\{k>k_{\text {Hopf }}\right\}$ to guarantee the existence and the stability of the pseudo-equilibrium $\widetilde{\mathbf{x}}_{-}$;

(ii) $k$ must be chosen sufficiently far away of $k_{\text {Hopf }}$, making as big as possible the uLC around the pseudo-equilibrium $\widetilde{\mathbf{x}}_{-}$.

Adopting these practical rules, we can guarantee the existence and stability of the operating point choosing, for instance, $k=1.5$ and assuming variations of parameters $a$ and $d$ as long as these values belong to regions III and IV in Fig. 16. These two regions are the safe operating region for the proposed SMC.

\section{Conclusions}

A useful methodology based on bifurcation theory to determine the stability and robustness of SMC has been illustrated through a realistic problem: a DBC controlled by a washout-SMC operating in an islanding DC microgrid. New concepts have been exploited; in particular, the de-singularized sliding vector field and its dimensional reduction via projection have been shown to be crucial for alleviate the computational effort for the analysis.

The nonlinear stability analysis performed in this work allow us to determine the safe parameter region for the robust system operation under load (parameter $a$ ) and power (parameter $d$ ) changes. This information can be summarized in bifurcation diagrams leading to practical rules for choosing the control parameter $k$ in order to achieve a robust SMC design.

In looking for stability conditions, we are of course disregarding practical aspects as, for instance, the use of a hysteresis mechanism to constraint the maximum switching frequency that, certainly, will increase the complexity of this analysis. Also, startup procedures to pass from initial conditions to the attraction basin of the operating point should be taken into account. Anyway, the bifurcation analysis, here developed, must not be underestimated since leads to a considerable insight about the dynamics of sliding mode controllers and how to modify it. 


\section{Acknowledgments}

This work was supported by the Coordenadoria de Aperfeicoamento de Pessoal de nivel Superior (CAPES), Brazil. L. Benadero was supported by the Spanish Ministerio de Ciencia e Innovación under grant DPI201347293-R and E. Ponce by MINECO/FEDER grant MTM2012-31821 and by the Consejería de Economía, Innovación, Ciencia y Empleo de la Junta de Andalucía under grant P12-FQM-1658.

\section{References}

Boroyevich, D., Cvetkovic, I., Dong Dong, Burgos, R., Fei Wang \& Lee, F. [2010] "Future electronic power distribution systems a contemplative view", 12th International Conference on Optimization of Electrical and Electronic Equipment, pp. 1369-1380.

Colombo, A., di Bernardo, M., Fossas, E. \& Jeffrey, M. R. [2010] "Teixeira singularities in 3D switched feedback control systems," Systems \& Control Letters, 59, pp. 615-622, 2010.

di Bernardo, M., Nordmark, A. \& Olivar, G. "Discontinuity-induced bifurcations of equilibria in piecewisesmooth dynamical systems," Physica D, 237, pp. 119-136, 2008.

di Bernardo, M. Pagano, D. J. \& Ponce, E. [2008] "Nonhyperbolic boundary equilibrium bifurcations in planar Filippov systems: A case study approach," Int. J. Bifurcation and Chaos, 18, pp. 1377-1392, 2008.

Emadi, A., Khaligh, A., Rivetta, C.H. \& Williamson, G.A. [2006] "Constant power loads and negative impedance instability in automotive systems: definition, modeling, stability, and control of power electronic converters and motor drives," IEEE Trans. on Vehicular Technology, vol.55, pp.1112-1125.

Filippov, A. F., [1988] Differential Equations with Discontinuous Righthand Sides, (The Netherlands: Kluwer Academic).

R. Haroun, A. Cid-Pastor, A. El Aroudi, and L. Martinez- Salamero, [2014] "Synthesis of canonical elements for power processing in dc distribution systems using cascaded converters and sliding-mode control", IEEE Trans. on Power Electronics, vol. 29, no. 3, pp. 1366-1381, March 2014.

R. Haroun, A. El Aroudi, A. Cid-Pastor, G. Garica, C. Olalla, and L. Martinez-Salamero, [2015] "Impedance matching in photovoltaic systems using cascaded boost converters and sliding mode control," IEEE Trans. on Power Electronics, vol. 30, no. 6, pp. 3185-3199.

Jeffrey, M. R. \& Colombo, A. [2009] "The Two-Fold Singularity of Discontinuous Vector Fields", SIAM J. Appl. Dyn. Syst., 8, pp. 624-640.

Kwasinski, A. \& Onwuchekwa, C., [2011] "Dynamic Behavior and Stabilization of dc Micro-grids with Instantaneous Constant-Power Loads," IEEE Trans. on Power Electronics, 26, pp. 822-834.

Kuznetsov, Y. A., Rinaldi, S. \& Gragnani, A. [2003] "One-Parameter Bifurcations in Planar Filippov Systems", Int. J. Bifurcation and Chaos, 13, pp. 2157-2188.

Lee, H. C. \& Abed, E. H. [1991] "Washout filter in the bifurcation control of high alpha flight dynamics," Proc. American Control Conference, 1, pp. 206-211.

Liutanakul, P., Awan, A. B., Pierfederici, S., Nahid-Mobarakeh, B. \& Meibody-Tabar, F. [2010] "Linear Stabilization of a DC Bus Supplying a Constant Power Load: A General Design Approach," IEEE Trans. on Power Electronics, 25, pp. 475-488.

Pagano, D. J., Ponce, E. \& Torres, F. [2011] "On Double Boundary Equilibrium Bifurcations in Piecewise Smooth Planar Systems," Qual. Theory Dyn. Syst. 10, pp. 277-301.

Stramosk, V., Benadero, L., Pagano, D. J. \& Ponce, E. [2013] "Sliding Mode Control of Interconnected Power Electronic Converters in DC Microgrids," $39^{\text {th }}$ Annual Conference IEEE Industrial Electronics Society, Vienna, pp. 8377-8382.

Ponce, E. \& Pagano, D. J. [2011] "Sliding Dynamics Bifurcations in the Control of Boost Converters," $18^{\text {th }}$ IFAC World Congress, Milano, Italy, pp. 13293-13298.

Tahim, A., Pagano, D. J. \& Ponce, E. [2012] "Nonlinear Control of Boost Bidirectional Converters in Stand-alone de Microgrids", 51 ${ }^{\text {st }}$ IEEE Conference on Decision and Control, Maui, USA, pp. 30683073.

Teixeira, M. A. [1990] "Stability Conditions for Discontinuous Vector Fields", J. Diff. Eqs., 88, pp. 15-29. 
Wang, H. and E. H. Abed. [1995] "Bifurcation control of a chaotic system," [1995] Automatica, 31, pp. $1213-1226$.

Xiong, X., Tse, C. K. \& Ruan, X. [2013] "Bifurcation Analysis of Standalone Photovoltaic-Battery Hybrid Power System," IEEE Trans. on Circuits and Systems-I: regular papers, 60, pp. 1354-1365.

Xiong, X., Tse, C. K. \& Ruan, X. [2013] "Smooth and Nonsmoooth Bifurcations in Multi-structure Multioperating-mode Hybrid Power Systems," Int. J. Bifurcation and Chaos, 23, 1350094 (12 pages). 